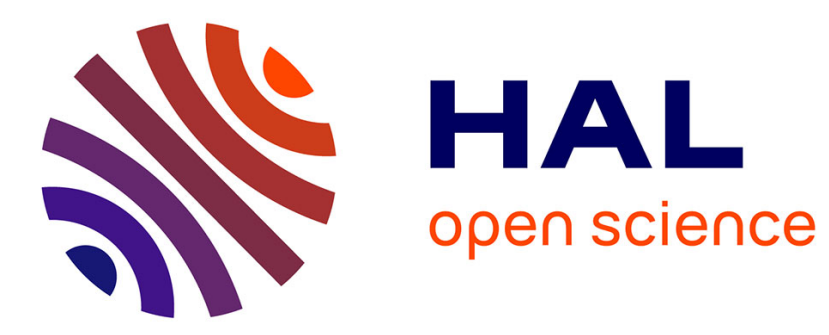

\title{
CLT for Lipschitz-Killing curvatures of excursion sets of Gaussian random fields
}

\author{
Marie Kratz, Sreekar Vadlamani
}

\section{To cite this version:}

Marie Kratz, Sreekar Vadlamani. CLT for Lipschitz-Killing curvatures of excursion sets of Gaussian random fields. 2016. hal-01373091

\section{HAL Id: hal-01373091 \\ https://essec.hal.science/hal-01373091}

Preprint submitted on 28 Sep 2016

HAL is a multi-disciplinary open access archive for the deposit and dissemination of scientific research documents, whether they are published or not. The documents may come from teaching and research institutions in France or abroad, or from public or private research centers.
L'archive ouverte pluridisciplinaire HAL, est destinée au dépôt et à la diffusion de documents scientifiques de niveau recherche, publiés ou non, émanant des établissements d'enseignement et de recherche français ou étrangers, des laboratoires publics ou privés. 


\title{
I \\ ESSEC \\ BUSINESS SCHOOL
}

CLT FOR LIPSCHITZ-KILLING CURVATURES OF EXCURSION SETS OF GAUSSIAN RANDOM FIELDS

\author{
RESEARCH CENTER \\ MARIE KRATZ, SREEKAR VADLAMANI \\ ESSEC WORKING PAPER 1615
}

AUGUST 2016 


\title{
CLT for Lipschitz-Killing curvatures of excursion sets of Gaussian random fields
}

\author{
Marie $\mathrm{Kratz}^{*}$ and Sreekar Vadlamani ${ }^{\dagger}$
}

August 5, 2016

\begin{abstract}
Our interest in this paper is to explore limit theorems for various geometric functionals of excursion sets of isotropic Gaussian random fields. In the past, limit theorems have been proven for various geometric functionals of excursion sets/sojourn times ( see $[4,13,14,18,22,25]$ for a sample of works in such settings). The most recent addition being [6] where a central limit theorem (CLT) for Euler-Poincaré characteristic of the excursions set of a Gaussian random field is proven under appropriate conditions. In this paper, we obtain a CLT for some global geometric functionals, called the LipschitzKilling curvatures of excursion sets of Gaussian random fields in an appropriate setting.

Keywords: chaos expansion, CLT, excursion sets, Gaussian fields, Lipschitz-Killing curvatures.
\end{abstract}

MSC2010: 60F05, 60G15, 60G60, 60G10, 60D05, 53C65, 14M15

\section{Introduction and main result}

There has been recently a surge in interest in understanding the geometry of random sets. In particular, there have been recent works on limit theorems of geometric functionals of random sets coming from discrete type models arising from various point processes (see [5], and references therein), or from models of smooth random fields [6, 14, 16, 17, 22, 25].

The object of this paper is to go further, and provide asymptotic distributions for some global geometric characteristics of the excursion sets of random fields as the parameter space is allowed to grow to infinity.

More precisely, let $f$ be a random field defined on $\mathbb{R}^{d}$, and let $\mathcal{T}$ be a $d$-dimensional box $[-T, T]^{d}$ We shall be considering the restriction of $f$ to the subset $\mathcal{T}$, and accordingly define

*ESSEC Business School, CREAR, Paris, France; E-mail: kratz@essec.edu

${ }^{\dagger}$ TIFR-Center for Applicable Mathematics (CAM), Bangalore, India; E-mail: sreekar@tifrbng.res.in 
the excursion set of $f$ over a threshold $u$, denoted by $A_{u}(f ; \mathcal{T})$, as

$$
A_{u}(f ; \mathcal{T})=\{x \in \mathcal{T}: f(x) \geq u\}
$$

Our interest, in this paper, is to study the distributional aspects of Lipschitz-Killing curvatures of the sets $A_{u}(f ; \mathcal{T})$.

The Lipschitz-Killing curvatures (LKCs) of a d-dimensional Whitney stratified manifold ${ }^{1}$ $M$ are $(d+1)$ integral geometric functionals $\left\{\mathcal{L}_{k}(M)\right\}_{k=0}^{d}$, with $\mathcal{L}_{0}(M)$ the Euler-Poincaré characteristic of the set $M$, and $\mathcal{L}_{d}(M)$ the $d$-dimensional Hausdorff measure of $M$. Though, for $k=1, \ldots, d-1$, the $\mathcal{L}_{k}(M)$ do not have such clear interpretation, the scaling property ${ }^{2}$ of the LKCs can be used to interpret the $k$-th LKC as a $k$-dimensional measure. This result not only underlines the importance of the LKCs, but also characterises them with their additive and scaling property together with the rigid motion invariance.

One of the most important results in convex geometry is the Hadwiger's characterisation theorem, which, in simple terms, states that the LKCs form a basis for all finitely additive, monotone and rigid motion invariant valuations defined on the the collection of basic complexes (cf. [1, 10]).

The main result of this paper provides a CLT for LKCs of $A_{u}(f ; \mathcal{T})$ of Gaussian random fields:

THEOREM 1.1 Let $\mathcal{T}$ be as defined above, and $f$ be a mean zero, unit variance, isotropic Gaussian random field defined on $\mathbb{R}^{d}$ with $C^{3}$ trajectories. Then, under some standard regularity assumptions on $f$ as stated in (H1), (H2) and (H3), we have

$$
\frac{\mathcal{L}_{k}\left(A_{u}(f ; \mathcal{T})\right)-\mathbb{E}\left(\mathcal{L}_{k}\left(A_{u}(f ; \mathcal{T})\right)\right)}{|\mathcal{T}|^{1 / 2}} \rightarrow N\left(0, \sigma_{k}^{2}(u)\right), \text { as } \mathcal{T} \rightarrow \mathbb{R}^{d}
$$

for $k=0, \ldots, d$, where by $\mathcal{T} \rightarrow \mathbb{R}^{d}$, we mean $T \rightarrow \infty$.

REMARK 1.1 We note here that the specific cases corresponding to $k=0$ and $k=d$ have already been dealt with in [6] and [22], respectively.

We shall adopt the now standard approach of projecting Gaussian functionals of interest onto the Itô-Wiener chaos, then use the Breuer-Major type of theorem to conclude our main result. This approach has been developed in [14] to obtain CLT for general level functionals of $\left(f, \partial f, \partial^{2} f\right)$ in dimension 1 , then extended to dimension 2 (see [11] for a general review on the topic). As applications, we got back CLTs for the number of crossings of $f$, a result first obtained by Slud with an alternative method (see [23, 24]), for the number of local maxima ([13]), for the sojourn time of $f$ in some interval ([14]), and also for the length of a level curve of a 2-dimensional Gaussian field ([14]). Note that in these papers, the last step of the method was to approximate $f$ with an $m$-dependent process ([4]) in order to conclude to the CLT. In fact, this step can be removed, and simplifies using what is now called the SteinMalliavin method to conclude Breuer-Major types of theorem, as documented in [19]. Based

\footnotetext{
${ }^{1}$ For more details on this, and more, we refer the reader to [1].

${ }^{2}$ For any $\lambda>0$, we have $\mathcal{L}_{k}(\lambda M)=\lambda^{k} \mathcal{L}_{k}(M)$, where $\lambda M=\{\lambda x: x \in M\}$.
} 
on this general approach, CLTs have been proved recently when considering a $d$-dimensional Gaussian random field $f$ by Pham [22] for the sojourn time of $f$, and by Estrade and León [6] for the Euler-Poincaré characteristic (EPC) of the excursion set of $f$. Note that EPC shares strikingly similar integral representation as the number of level crossings in terms of functional of $f$, but for dimension $d$. Largely, the sketch of the proof for CLTs and the main technical steps remain the same as in [14] when dealing with level functionals of a $d$-dimensional Gaussian field $f$, with $d>2$; the difficulty lies in finding a way to avoid explicit computations. Hence the main and crucial contribution in [6] has been to come up with a neat trick to circumvent this difficulty, proving that the order of the variance of the EPC for $f$ restricted to any subspace of $\mathcal{T}$ is less than $|\mathcal{T}|$, hence is negligible in the limit as $\mathcal{T}$ grows to $\mathbb{R}^{d}$.

We are going to build on these works, in order to obtain a CLT not only for EPC but for any LKC. The difficulty here is to develop similar techniques when working on $A_{u}\left(f ; \mathcal{T} \cap V^{*}\right)$ for any $k$-dimensional affine subspace $V$ of $\mathbb{R}^{d}$.

The structure of the paper is as follows. Throughout this paper, we work with isotropic Gaussian random fields. We begin in Section 2 with setting the notation, and the necessary background for the analysis to follow in later sections. In Section 2.1, we recall the basics of the expansions of Gaussian functionals using the multiple Wiener-Itô integrals. Next, in Section 2.2, we define the Lipschitz-Killing curvatures, and also state the Crofton formula that provides a relationship between various LKCs; it is going to be a crucial element in the proof of our main result. Section 2.3 is devoted to discuss the integral representation of EulerPoincaré characteristic of excursion sets of any random field via the expectation metatheorem. Finally, precise setup for the problems, and the assumptions, in particular on the covariance structure of the random field $f$, is listed in Section 2.4. In Section 3, we develop the proof of the main result, Theorem 1.1, using the standard sketch given in three main steps. First we prove that the functional of interest is square-integrable (Section 3.1) and obtain its Hermite expansion in Section 3.2. Then we prove that the limiting variance is bounded away from zero and infinity in Section 3.3. Finally, in Section 3.4, we give an extension of Breuer-Major theorem to affine Grassmannian case to conclude to the Gaussianity of the limiting distribution.

\section{Preliminaries}

\subsection{Itô-Wiener chaos expansions}

Hermite polynomial expansions or Multiple Wiener-Itô Integrals (MWI) are a powerful tool to approximate and study nonlinear functionals of stationary Gaussian fields (see [6], [11], or [14] for details).

Formally, let $Z$ be a $m$-dimensional standard Gaussian random vector, and let $L^{2}(Z)$ be the set of all real square integrable functionals of $Z$. In short, giving a Hermite expansion is a way to approximate elements from $L^{2}(Z)$ by a series of Hermite polynomials. More precisely, for $\underline{n} \in(\mathbb{N} \cup\{0\})^{m}$, define $\mathcal{H}_{\underline{n}}(Z)=\prod_{i=1}^{m} H_{n_{i}}\left(Z_{i}\right)$, and set $\mathcal{H}_{q}$ as the linear span 
of $\left\{\mathcal{H}_{\underline{n}}(Z):|\underline{n}|=\sum_{i=1}^{m} n_{i}=q\right\}$. Then,

$$
L^{2}(Z)=\bigoplus_{q \geq 0} \mathcal{H}_{q}
$$

For more details regarding Hermite expansions, and their applications to study functionals of Gaussian random fields, we refer the reader to [4, 19, 29].

The above can also be written in a more abstract setting of multiple Wiener integrals, for which let us begin with an orthonormal system $\left\{\varrho_{i}\right\}_{i \geq 1}$ of $L^{2}\left(\mathbb{R}^{m}\right)$. Writing $W$ for complex Brownian measure on $\mathbb{R}^{m}$, let us define $\xi_{i}=\int_{\mathbb{R}^{m}} \varrho_{i}(\lambda) W(d \lambda)$. Clearly, $\left\{\xi_{i}\right\}$ form a sequence of i.i.d. standard normal random variables. Now for a fixed $\underline{n} \in(\mathbb{N} \cup\{0\})^{q}$, and $p_{1}, \ldots, p_{q} \in \mathbb{N}$ define $H_{\underline{n}}\left(\xi_{p_{1}}, \ldots, \xi_{p_{q}}\right)=\prod_{i=1}^{q} H_{n_{i}}\left(\xi_{p_{i}}\right)$. Then (see [21])

$$
\begin{aligned}
H_{\underline{n}}\left(\xi_{p_{1}}, \ldots, \xi_{p_{q}}\right) & =\int_{\mathbb{R}^{m q}}\left(\varrho_{p_{1}}^{\otimes n_{1}} \otimes \cdots \otimes \varrho_{p_{q}}^{\otimes n_{q}}\right)\left(\lambda_{1}, \ldots, \lambda_{q}\right) W\left(d \lambda_{1}\right) \cdots W\left(d \lambda_{q}\right) \\
& \triangleq I_{q}\left(\varrho_{p_{1}}^{\otimes n_{1}} \otimes \cdots \otimes \varrho_{p_{q}}^{\otimes n_{q}}\right)
\end{aligned}
$$

where $I_{q}$ denotes multiple Wiener integral. A decomposition, similar to (2), holds true for all square integrable functionals of $W$, and is called the Itô-Wiener chaos. We refer the reader to [21] for complete details.

REMARK 2.1 The above Hermite expansion, at the first look, seems to work for single Gaussian element. However, using [19, Proposition 7.2.3] one can obtain similar Hermite expansions for functionals of stationary Gaussian fields.

\subsection{Lipschitz Killing curvatures and the Crofton formula}

There are a number of ways to define Lipschitz-Killing curvatures, but perhaps the easiest is via the so-called Weyl's tube formula (see $[9,28]$ for the first hand account of this formula). In order to state the tube formula, let $M$ be an $m$-dimensional manifold with positive reach (cf. [1]) embedded in $\mathbb{R}^{n}$ which is endowed with the canonical Riemannian structure on $\mathbb{R}^{n}$. Then, writing $\|\cdot\|$ as the standard Euclidean norm on $\mathbb{R}^{n}$, the tube of radius $\rho$ around $M$ is defined as

$$
\operatorname{Tube}(M, \rho)=\left\{x \in \mathbb{R}^{n}: \inf _{y \in M}\|x-y\| \leq \rho\right\} .
$$

Then according to Weyl's tube formula (see [1]), the Lebesgue volume of so constructed tube, for small enough $\rho$, is given by

$$
\lambda_{n}(\operatorname{Tube}(M, \rho))=\sum_{j=0}^{m} \rho^{n-j} \omega_{n-j} \mathcal{L}_{j}(M),
$$

where $\omega_{n-j}$ is the volume of the $(n-j)$-dimensional unit ball in $\mathbb{R}^{n-j}$, and $\mathcal{L}_{j}(M)$ is the $j$-th LKC of $M$. 
Although, it may appear from the definition above that the $\mathcal{L}_{j}$ depend on the embedding of $M$ in $\mathbb{R}^{n}$, in fact, the $\mathcal{L}_{j}(M)$ are intrinsic, and so are independent of the ambient space.

Apart from their appearance in the tube formula (5), there are, at least, two more ways in which to define the LKCs (see [1]).

Borrowing the notations from [1], let $\operatorname{Graff}(d, k)$ be the affine Grassmannian of all $k$ dimensional affine subspaces of $\mathbb{R}^{d}$, and $\mathbf{G r}(d, k)$ be the set of all $k$-dimensional linear subspaces of $\mathbb{R}^{d}$.

Let $M$ be a compact subset of $\mathbb{R}^{d}$ and $V^{*} \in \operatorname{Graff}(d, k)$. Then writing

$$
M_{V^{*}} \text { for }\left(M \cap V^{*}\right),
$$

and setting $\lambda_{k}^{d}$ to be the appropriate, normalised measure on $\operatorname{Graff}(d, k)$, and also

$$
\left[\begin{array}{c}
m \\
n
\end{array}\right]=\frac{\omega_{m}}{\omega_{n} \omega_{m-n}}\left(\begin{array}{c}
m \\
n
\end{array}\right)
$$

we have the Crofton formula:

$$
\int_{\operatorname{Graff}(d, k)} \mathcal{L}_{j}\left(M_{V^{*}}\right) d \lambda_{k}^{d}\left(V^{*}\right)=\left[\begin{array}{c}
d-k+j \\
j
\end{array}\right] \mathcal{L}_{d-k+j}(M)
$$

whenever $M$ is tame and a Whitney stratified space (cf [1]).

Setting $j=0$ in the above equation (6) gives back the Hadwiger formula

$$
\int_{\operatorname{Graff}(d, k)} \mathcal{L}_{0}\left(M_{V^{*}}\right) d \lambda_{k}^{d}\left(V^{*}\right)=\mathcal{L}_{d-k}(M)
$$

which we shall use to generate all the LKCs given the Euler-Poincaré characteristic of all the slices $M_{V^{*}}$.

Another interesting case is when we set $j=k$ in (6); we obtain

$$
\int_{\operatorname{Graff}(d, k)}\left|M_{V^{*}}\right| d \lambda_{k}^{d}\left(V^{*}\right)=\left[\begin{array}{l}
d \\
k
\end{array}\right] \mathcal{L}_{d}(M)
$$

where $\left|M_{V^{*}}\right|$ is the $k$-dimensional Hausdorff measure of the set $M_{V^{*}}$.

\subsection{Euler-Poincaré characteristic and other LKCs of excursion sets}

Let $\mathcal{T}$ be a compact, tame and Whitney stratified subset of $\mathbb{R}^{d}$. For any fixed $V^{*} \in$ $\operatorname{Graff}(d, k)$, set $\partial_{l} \mathcal{T}_{V^{*}}$ as the $l$-dimensional boundary of $\mathcal{T}_{V^{*}}$. Assume $f$ be a smooth Gaussian random field, then using the standard Morse theory (see [1, Chapter 9]), we can write

$$
\mathcal{L}_{0}\left(A_{u}\left(f ; \mathcal{T}_{V^{*}}\right)\right)=\sum_{l=0}^{k} \sum_{J \in \partial_{l} \mathcal{T}_{V^{*}}} \phi_{l}(J)
$$


whenever $\mathcal{T}$ is tame and a Whitney stratified space (cf. [1]), where,

$$
\phi_{l}(J)=\sum_{j=0}^{l}(-1)^{j} \#\left\{t \in J: f(t) \geq u, \nabla_{J} f(t)=0, \operatorname{index}\left(\nabla_{J}^{2} f(t)\right)=l-j\right\},
$$

with $\nabla_{J} f$ and $\nabla_{J}^{2} f$ representing restrictions of the usual gradient $\nabla f$ and Hessian $\nabla^{2} f$ onto $J \in \partial_{l} \mathcal{T}_{V^{*}}$.

Applying Theorem 11.2.3 of [1], the above equation can be rewritten as

$$
\phi_{l}(J)=(-1)^{l} \int_{\mathcal{T}_{V^{*}}} \delta\left(\nabla_{J} f(t)\right) \mathbb{I}_{\{f(t) \geq u\}} \operatorname{det}\left(\nabla_{J}^{2} f(t)\right) d t
$$

almost surely and in $L^{2}$, where $\delta$ is the Dirac delta at 0 defined on $\mathbb{R}^{d}$.

Remark 2.2 (Parametrization of $\operatorname{GrafF}(d, k)$ )

Note that $\operatorname{Graff}(d, k)$ can be parametrized as $\operatorname{Gr}(d, k) \times \mathbb{R}^{d-k}$. Furthermore, we shall identify $G r(d, k)$ with the set of all $k \times d$ matrices whose rows are orthonormal vectors in $\mathbb{R}^{d}$.

Writing $V$ as the matrix whose rows are $k$-orthonormal vectors spanning the linear space obtained by the parallel translate of $V^{*}$,

$$
\phi_{k}\left(\partial_{k} \mathcal{T}_{V^{*}}\right)=(-1)^{k} \int_{\mathcal{T}_{V^{*}}} \delta(V \nabla f(t)) \mathbb{I}_{\{f(t) \geq u\}} \operatorname{det}\left(V \nabla^{2} f(t) V^{T}\right) d t
$$

Note: For any $V^{*} \in \operatorname{Graff}(d, k)$, we shall denote $V$ for the matrix whose rows are $k$ orthonormal vectors spanning the linear space obtained by the parallel translate of $V^{*}$, and we shall use the same $V$ to denote the element in $\operatorname{Gr}(d, k)$ that corresponds to the $k$ dimensional linear space spanned by the rows of the matrix $V$.

We shall combine equations (7) and (9) to express all other LKCs in terms of the EulerPoincaré characteristic of $A_{u}\left(f ; \mathcal{T}_{V^{*}}\right)$. Formally,

$$
\mathcal{L}_{d-k}\left(A_{u}(f ; \mathcal{T})\right)=\int_{\operatorname{Graff}(d, k)} \mathcal{L}_{0}\left(A_{u}\left(f ; \mathcal{T}_{V^{*}}\right)\right) d \lambda_{k}^{d}\left(V^{*}\right)
$$

\subsection{Setup for the problem and assumptions}

In this paper, we consider $f$ a mean zero, isotropic, real valued Gaussian random field defined on $\mathbb{R}^{d}$ with $C^{3}$ trajectories. The assumption of isotropy means that the covariance of the Gaussian random field has a special form

$$
\mathbb{E}[f(x) f(y)]=r(|x-y|), \quad \forall x, y \in \mathbb{R}^{d}
$$

for some function $r: \mathbb{R}_{+} \rightarrow \mathbb{R}$. Without loss of generality, we shall assume $r(0)=1$. 
We denote the partial derivatives of order $n$ of any function $g$ defined on $\mathbb{R}^{d}$ as

$$
g^{\left(i_{1} \cdots i_{n}\right)}(t)=\frac{\partial^{n}}{\partial t_{i_{1}} \cdots \partial t_{i_{n}}} g(t) .
$$

We introduce the gradient $\nabla f(x)$ and Hessian $\left.\nabla^{2} f(x)\right)$ of $f$, and recall that due to isotropy $\nabla f(x)$ and $\left(f(x), \nabla^{2} f(x)\right)$ are independent for every fixed $x$ (in fact, stationarity suffices to conclude the same). Thus, the covariance function of $\left(\nabla f(x), f(x), \nabla^{2} f(x)\right)$ can be expressed as a block diagonal matrix for each fixed $x$. We denote the covariance matrix of $\left(\nabla f(x), f(x), \nabla^{2} f(x)\right)$ as

$$
\Sigma=\left(\begin{array}{cc}
\Sigma_{1} & 0 \\
0 & \Sigma_{2}
\end{array}\right)
$$

where $\Sigma_{1}$ and $\Sigma_{2}$ are the covariance matrices of $\nabla f$, and $\left(f, \nabla^{2} f\right)$, respectively. Notice that since $\left(\nabla f(x), f(x), \nabla^{2} f(x)\right)$ is a $(d+1+d(d+1) / 2)$ dimensional vector, the corresponding covariance matrix is a square matrix of order $D \times D$, where

$$
D=d+1+d(d+1) / 2 .
$$

Simple linear algebraic considerations imply that there exists a $D \times D$ matrix $\Lambda$ such that $\Lambda \Lambda^{T}=\Sigma$. Then we define a new field $Z=\left(Z(x), x \in \mathbb{R}^{d}\right)$ by

$$
Z(x)=\Lambda^{-1}\left(\nabla f(x), \nabla^{2} f(x), f(x)\right) .
$$

Let us denote its covariance function by

$$
\gamma=\left(\gamma_{i j}(.)\right)_{1 \leq i, j \leq D} \quad \text { with } \gamma_{i j}(h)=\operatorname{cov}\left(Z_{i}(x), Z_{j}(x+h)\right) .
$$

Note here that we have implicitly used the fact that various derivatives of a stationary Gaussian random field are themselves stationary Gaussian random fields (cf. [1, Chapter $5])$.

Note that we can write $Z$ as $Z(x)=\left(Z^{(1)}(x), Z^{(2)}(x)\right)$ so that

$$
Z(x)=\left(Z^{(1)}(x), Z^{(2)}(x)\right) \sim \mathcal{N}\left(\underline{0}, I_{D}\right), \quad I_{D} \text { being the identity in } \mathbb{R}^{D} .
$$

We need more assumptions on $f$ to ensure that various LKCs of the excursion set $A_{u}(f ; \mathcal{T})$ of $f$ over a threshold $u$, are indeed square integrable, and that they satisfy a CLT as $\mathcal{T} \rightarrow \mathbb{R}^{d}$.

The required assumptions are rather standard when looking for CLT of non linear functionals of stationary Gaussian random fields, such as number of crossings [14], curve length [14], EPC [6], sojourn time [22], etc.

(H1) Geman type condition: We shall assume that the covariance function $r \in C^{4}(\mathcal{T})$, and that the function $\frac{1}{\|t\|^{2}}\left(\nabla^{2} r(t)-r^{(i i)}(0) I_{d}\right)$ defined on $\mathbb{R}^{d}$, is bounded near $t=0$, where we recall that $r^{(i i)}(0)=-\operatorname{var}\left(\nabla_{i} f(x)\right)$.

(H1) is simply the higher dimensional analog of Geman's condition ([7]), which is needed to prove that the functional of interest is in $L^{2}$. When $d=1$, it is known to be a necessary and sufficient condition to obtain the $L^{2}$ convergence of the number of crossings of any threshold (see [15]). 
Observe that this condition (H1) is satisfied whenever the underlying random field is 'smooth' enough, with $C^{3}$ sample paths ${ }^{3}$. Hence, for simplicity, we will assume now on that $\mathbf{f}$ is $\mathbf{C}^{3}$.

(H2) Arcones type condition: For the covariance function $\gamma(\cdot)$ of the joint field $(Z(x))_{x \in \mathcal{T}}$, we assume that there exists an integrable $\psi$ on $\mathbb{R}^{d}$ satisfying

$$
\psi(t) \underset{\|t\| \rightarrow+\infty}{\longrightarrow} 0,
$$

such that

$$
\max _{1 \leq i, j \leq D}\left|(\gamma(x))_{i j}\right| \leq K \psi(x), \quad \text { for some } K>0 .
$$

This condition is crucial in ensuring the finiteness of the limiting variance of the considered functionals. As already noted in [14] and [6], it implies in particular the existence of the spectral density, and that $r \in L^{q}\left(\mathbb{R}^{d}\right), q \geq 1$.

(H3) The spectral density, denoted $h$, of the covariance function corresponding to the field $\left(\nabla f, \nabla^{2} f, f\right)$ satisfies $h(0)>0$.

This condition is equivalent to appropriate decay conditions on various (partial) derivatives of the covariance function $r$ (cf. [14]), and will be needed when proving that the asymptotic variance obtained in the CLT is non 0.

\section{Proof of Theorem 1.1}

Recall that the key argument in [6] to prove the CLT for the Euler-Poincaré characteristics of the excursion set is to consider only the highest dimensional term in (9), dropping all lower dimensional terms, and proving later that the contribution from the lower dimensional terms is negligible under the volume scaling. We will also use this argument.

Let us begin with

$$
\begin{aligned}
& \mathcal{L}_{d-k}\left(A_{u}(f ; \mathcal{T})\right) \\
= & \int_{\operatorname{Graff}(d, k)} \sum_{l=0}^{k}(-1)^{l} \sum_{J \in \partial_{l} \mathcal{T}_{V}} \int_{J} \mathbb{I}_{(f(x) \geq u)} \delta\left(\nabla_{J} f(x)\right) \operatorname{det}\left(\nabla_{J}^{2} f(x)\right) d x d \lambda_{k}^{d}(V) \\
= & \sum_{l=0}^{k}(-1)^{l} \int_{\operatorname{Graff}(d, k)} \sum_{J \in \partial_{l} \mathcal{T}_{V}} \int_{J} \mathbb{I}_{(f(x) \geq u)} \delta\left(\nabla_{J} f(x)\right) \operatorname{det}\left(\nabla_{J}^{2} f(x)\right) d x d \lambda_{k}^{d}(V) \\
= & \sum_{l=0}^{k} \mathcal{L}_{d-k, l}\left(A_{u}(f ; \mathcal{T})\right)
\end{aligned}
$$

where

$$
\mathcal{L}_{d-k, l}\left(A_{u}(f ; \mathcal{T})\right)=(-1)^{l} \int_{\operatorname{Graff}(d, k)} \sum_{J \in \partial_{l} \mathcal{T}_{V}} \int_{J} \mathbb{I}_{(f(x) \geq u)} \delta\left(\nabla_{J} f(x)\right) \operatorname{det}\left(\nabla_{J}^{2} f(x)\right) d x d \lambda_{k}^{d}(V)
$$

\footnotetext{
${ }^{3}$ We refer to [3] for further discussion on connections between analytical assumptions and regularity conditions for Gaussian random fields
} 
We shall consider only $\mathcal{L}_{d-k, k}\left(A_{u}(f ; \mathcal{T})\right)$, and prove that, after appropriate normalisation, it exhibits a central limit theorem. Thereafter, the same arguments can be pieced together to conclude that, under the same scaling, $\mathcal{L}_{d-k, l}\left(A_{u}(f ; \mathcal{T})\right)$ converge to 0 whenever $l<k$.

As spelt out in the introduction, our proof has three major steps:

1. to prove that the functional of interest is square integrable, and thereby obtain its Hermite type expansion;

2. to prove that the limiting variance is bounded away from zero and infinity;

3. to use the Stein-Malliavin method for Breuer-Major type functionals to conclude to the Gaussianity of the limiting distribution.

We shall provide details of the aforementioned steps in the following subsections.

\subsection{Square integrability}

Let us recall (11), and write

$$
\mathcal{L}_{d-k, k}\left(A_{u}(f ; \mathcal{T})\right)=\int_{\operatorname{Graff}(d, k)} \phi_{k}\left(\partial_{k} \mathcal{T}_{V^{*}}\right) d \lambda_{k}^{d}\left(V^{*}\right) .
$$

Clearly, by Jensen's inequality,

$$
\mathbb{E}\left[\left(\mathcal{L}_{d-k, k}\left(A_{u}(f ; \mathcal{T})\right)\right)^{2}\right] \leq \int_{\operatorname{Graff}(d, k)} \mathbb{E}\left[\left(\phi_{k}\left(\partial_{k} \mathcal{T}_{V^{*}}\right)\right)^{2}\right] d \lambda_{k}^{d}\left(V^{*}\right) .
$$

We shall now focus on obtaining an appropriate upper bound for $\mathbb{E}\left[\left(\phi_{k}\left(\partial_{k} \mathcal{T}_{V^{*}}\right)\right)^{2}\right]$, which in turn shall imply the square integrability of $\mathcal{L}_{d-k, k}\left(A_{u}(f ; \mathcal{T})\right)$.

Using standard fare, notice that $\phi_{k}\left(\partial_{k} \mathcal{T}_{V^{*}}\right)$ can be bounded above by the cardinality of the set $\{t: V \nabla f=0\}$ that we denote by $N_{u}\left(\mathcal{T}_{V^{*}}\right)$. Then, as usual, we compute the second factorial moment of $N_{u}(\mathcal{T})$ and prove that it is finite to conclude the square integrability. We have, using [1, Corollary 11.5.2], or [3, Theorem 6.2]

$$
\begin{aligned}
& \mathbb{E}\left[N_{u}\left(\mathcal{T}_{V^{*}}\right)\left(N_{u}\left(\mathcal{T}_{V^{*}}\right)-1\right)\right]= \\
& \int_{\mathcal{T}_{V^{*}}^{2}} \mathbb{E}\left(\left|\operatorname{det}\left(V \nabla^{2} f\left(t_{1}\right) V^{T}\right) \operatorname{det}\left(V \nabla^{2} f\left(t_{2}\right) V^{T}\right)\right| \mid V \nabla f\left(t_{1}\right)=V \nabla f\left(t_{2}\right)=0\right) p_{V, t_{1}, t_{2}}(0,0) d t_{1} d t_{2}(21)
\end{aligned}
$$

where $\mathcal{T}_{V^{*}}^{2}=\mathcal{T}_{V^{*}} \times \mathcal{T}_{V^{*}} /\left\{t_{1} \in \mathcal{T}_{V^{*}}, t_{2} \in \mathcal{T}_{V^{*}}: t_{1}=t_{2}\right\}$, and $p_{V, t_{1}, t_{2}}(0,0)$ is the joint density 
of $\left(V \nabla f\left(t_{1}\right), V \nabla f\left(t_{2}\right)\right)$. Using stationarity, we can reduce the above integral to

$$
\begin{aligned}
& \mathbb{E}\left[N_{u}\left(\mathcal{T}_{V^{*}}\right)\left(N_{u}\left(\mathcal{T}_{V^{*}}\right)-1\right)\right]= \\
& \int_{\mathcal{T}} \mathbb{E}\left(\mid \operatorname{det}\left(V \nabla^{2} f\left(t_{1}\right) V^{T}\right) \operatorname{det}\left(V \nabla^{2} f\left(t_{1}-s\right) V^{T}\right) \| V \nabla f\left(t_{1}\right)=V \nabla f\left(t_{1}-s\right)=0\right) p_{V, t_{1}, t_{1}-s}(0,0) d s d t_{1} \\
& t_{1} \in \mathcal{T}_{V^{*}} \\
& s \in\left(\mathcal{T}_{V^{*}}-t_{1}\right) /\{0\} \\
& =\quad \int_{t_{1} \in \mathcal{T}_{V^{*}}} \mathbb{E}\left(\mid \operatorname{det}\left(V \nabla^{2} f(0) V^{T}\right) \operatorname{det}\left(V \nabla^{2} f(s) V^{T}\right) \| V \nabla f(0)=V \nabla f(s)=0\right) p_{V, 0, s}(0,0) d s d t_{1} \text {. } \\
& s \in\left(\mathcal{T}_{V^{*}}-t_{1}\right) /\{0\}
\end{aligned}
$$

Next, using stationarity and Cauchy-Schwarz inequality gives

$$
\begin{aligned}
& \mathbb{E}\left(\left|\operatorname{det}\left(V \nabla^{2} f(0) V^{T}\right) \operatorname{det}\left(V \nabla^{2} f(s) V^{T}\right)\right| \mid V \nabla f(0)=V \nabla f(s)=0\right) \\
\leq & \mathbb{E}\left(\left|\operatorname{det}\left(V \nabla^{2} f(0) V^{T}\right)\right|^{2} \mid V \nabla f(0)=V \nabla f(s)=0\right) .
\end{aligned}
$$

Invoking similar methods as set forth in [6] we can conclude that there exists a constant $C_{1}$ (independent of $T_{V^{*}}$ ) such that

$$
\mathbb{E}\left(\left|\operatorname{det}\left(V \nabla^{2} f(0) V^{T}\right)\right|^{2} \mid V \nabla f(0)=V \nabla f(s)=0\right) \leq C_{1}\|s\|^{2} .
$$

Next, notice that

$$
p_{V, 0, s}(0,0) \leq C_{2}\|s\|^{-k}
$$

where $C_{2}$ is a constant independent of $V$.

Combining equations (22) and (23) in the integral in (21) provides

$$
\mathbb{E}\left[N_{u}\left(\mathcal{T}_{V^{*}}\right)\left(N_{u}\left(\mathcal{T}_{V^{*}}\right)-1\right)\right] \leq C_{3} \int_{\substack{t_{1} \in \mathcal{T}_{V^{*}} \\ s \in\left(\mathcal{T}_{V^{*}}-t_{1}\right) /\{0\}}}\|s\|^{2-k} d s d t_{1}
$$

for some constant $C_{3} \in(0, \infty)$.

Observe that, using polar decomposition, one can uniformly bound the integral over the set $\left(\mathcal{T}_{V^{*}}-t_{1}\right) /\{0\}$, and therefore, we have

$$
\mathbb{E}\left[N_{u}\left(\mathcal{T}_{V^{*}}\right)\left(N_{u}\left(\mathcal{T}_{V^{*}}\right)-1\right)\right] \leq C_{4}|\mathcal{T}|^{2 / d}\left|\mathcal{T}_{V^{*}}\right|
$$

for some positive constant $C_{4}$ independent of the choice of $V^{*}$. Finally, using the standard Gaussian kinematic fundamental formula (cf.[1]) for the mean of $N_{u}\left(\mathcal{T}_{V^{*}}\right)$ and the above computations together with the Crofton formula, we can conclude that

$$
\mathbb{E}\left(\mathcal{L}_{d-k, k}\left(A_{u}(f ; \mathcal{T})\right)\right)^{2}<C|\mathcal{T}|^{\frac{2+d}{d}}
$$

for some large, but finite and positive constant $C$. 


\subsection{Hermite expansion}

Set $F(x)=\left(f_{1}(x), f_{2}(x)\right)$ with $f_{1}(x)=\nabla f(x)$ and $f_{2}(x)=\left(\nabla^{2} f(x), f(x)\right)$.

For $x \in \mathcal{T}$, recall (see Section 2.4) that we can factorize $\Sigma$ as $\Sigma=\Lambda \Lambda^{T}$, such that $\Lambda$ has a block diagonal form

$$
\Lambda=\left(\begin{array}{cc}
\Lambda_{1} & 0 \\
0 & \Lambda_{2}
\end{array}\right)
$$

where $\Lambda_{1}$ is the formal square root of $\Sigma_{1}$ and $\Lambda_{2}$ is a lower triangular matrix such that $\Lambda_{2} \Lambda_{2}^{T}=\Sigma_{2}$, respectively.

Using equation (11) and standard methods as of [6], we now obtain a Hermite expansion for $\phi_{k}\left(\partial_{k} \mathcal{T}_{V^{*}}\right)$. Define

$$
\begin{aligned}
G_{1}^{V}\left(f_{1}(x)\right) & =\delta(V \nabla f(x)) \\
G_{2, u}^{V}\left(f_{2}(x)\right) & =\mathbb{I}_{(f(x) \geq u)} \operatorname{det}\left(V \nabla^{2} f(x) V^{T}\right) .
\end{aligned}
$$

Clearly, for each fixed space point $x$, the functions $G_{1}$ and $G_{2}$ are independent. We shall obtain Hermite expansions for these two functions separately.

REMARK 3.1 Since $G_{1}$ is not a function, the way of obtaining a Hermite expansion goes through a limiting process. However, this process of approximation is clearly spelled out in many of previous works going as far back as [4], and hence we shall omit this step, and skip to the limit.

Formally, for $\underline{n} \in \mathbb{N}^{D}, D$ being defined in (14), set $\underline{n}=\left(\underline{n}_{1}, \underline{n}_{2}\right) \in \mathbb{N}^{d} \times \mathbb{N}^{D-d}$, then the square integrability implies that we have the following

$$
\begin{aligned}
& G_{1}^{V}\left(f_{1}(x)\right) \times G_{2, u}^{V}\left(f_{2}(x)\right) \\
& =\sum_{q=0}^{\infty} \sum_{\underline{n}^{(D)}: \sum_{i=1}^{D} n_{i}=q} c(\underline{n}, u, V, \Lambda) H_{\underline{n}_{1}}\left(Z^{(1)}(x)\right) H_{\underline{n}_{2}}\left(Z^{(2)}(x)\right)
\end{aligned}
$$

where the Hermite coefficients are given by

$$
\begin{aligned}
c(\underline{n}, u, V, \Lambda) & :=\frac{1}{\underline{n} !} \int_{\mathbb{R}^{D}} G_{u}^{V}(\Lambda \underline{y}) \prod_{i=1}^{D} H_{n_{i}}\left(y_{i}\right) \varphi_{D}(\underline{y}) d \underline{y} \\
& =c_{1}\left(\underline{n}_{1}, V, \Lambda_{1}\right) \times c_{2}\left(\underline{n}_{2}, u, V, \Lambda_{2}\right)
\end{aligned}
$$

writing $\varphi_{D}$ for the standard normal density in $D$-dimensions.

REMARK 3.2 It follows from the discussion of [1, Section 5.7], that the distribution of $\left(V \nabla f(x), V \nabla^{2} f(x) V^{T}\right)$ does not depend on the space point $x$ (due to stationarity) and the space $V$ (due to isotropy). Therefore, the coefficients $c_{1}\left(\underline{n}_{1}, V, \Lambda_{1}\right)$ and $c_{2}\left(\underline{n}_{2}, u, V, \Lambda_{2}\right)$ do not depend on $V$, which will help simplifying the proofs. 


\section{Computing $c_{1}$}

Observe that

$$
c_{1}\left(\underline{n}_{1}, V, \Lambda_{1}\right)=\frac{1}{\underline{n}_{1} !} \int_{\mathbb{R}^{d}} G_{1}^{V}\left(\Lambda_{1} \underline{y}_{1}\right) H_{\underline{n}_{1}}\left(\underline{y}_{1}\right) \varphi_{k}\left(\underline{y}_{1}\right) d \underline{y}_{1} .
$$

First, note that the integral is to be interpreted as a limit of integral of an appropriate approximation of $\delta$. Secondly, by Remark 3.2, we can choose a $V$ which suits our purpose.

In particular, one may define

$$
G_{1, \varepsilon}^{V}\left(\Lambda_{1} \underline{y}_{1}\right)=\frac{1}{\left(2 \pi \varepsilon^{2}\right)^{k / 2}} \exp \left(-\frac{1}{2 \varepsilon^{2}} \underline{y}_{1}^{T} \Lambda_{1}^{T} V^{T} V \Lambda_{1} \underline{y}_{1}\right),
$$

and thus define $c_{1}\left(\underline{n}_{1}, V, \Lambda_{1}\right)$ as an $L^{2}$-limit of

$$
c_{1}\left(\underline{n}_{1}, \varepsilon, V, \Lambda_{1}\right) \triangleq \frac{1}{\underline{n}_{1} !\left(2 \pi \varepsilon^{2}\right)^{k / 2}} \int_{\mathbb{R}^{d}} \exp \left(-\frac{1}{2 \varepsilon^{2}} \underline{y}_{1}^{T} \Lambda_{1}^{T} V^{T} V \Lambda_{1} \underline{y}_{1}\right) H_{\underline{n}_{1}}\left(\underline{y}_{1}\right) \varphi_{k}\left(\underline{y}_{1}\right) d \underline{y}_{1} .
$$

Noticing that the variance of $\nabla_{i} f(x)$ does not depend on the index $i$ due to isotropy, we conclude that $\Lambda_{1}=\sqrt{\lambda} I_{d}$ where $\lambda$ is the variance of $\nabla_{i} f(x)$. Therefore,

$$
c_{1}\left(\underline{n}_{1}, \varepsilon, V, \Lambda_{1}\right)=\frac{1}{\underline{n}_{1} !\left(2 \pi \varepsilon^{2}\right)^{k / 2}} \int_{\mathbb{R}^{d}} \exp \left(-\frac{1}{2 \varepsilon^{2}} \lambda \underline{y}_{1}^{T} V^{T} V \underline{y}_{1}\right) H_{\underline{n}_{1}}\left(\underline{y}_{1}\right) \varphi_{d}\left(\underline{y}_{1}\right) d \underline{y}_{1} .
$$

Equivalently,

$$
c_{1}\left(\underline{n}_{1}, \varepsilon, V, \Lambda_{1}\right)=\frac{\lambda^{-k / 2}}{\underline{n}_{1} !} \int_{\mathbb{R}^{d}} \frac{\lambda^{k / 2}}{\varepsilon^{k}} \varphi_{k}\left(\frac{1}{\varepsilon} \sqrt{\lambda} V \underline{y}_{1}\right) H_{\underline{n}_{1}}\left(\underline{y}_{1}\right) \varphi_{d}\left(\underline{y}_{1}\right) d \underline{y}_{1},
$$

where $\frac{\lambda^{k / 2}}{\varepsilon^{k}} \varphi_{k}\left(\sqrt{\lambda} \varepsilon V \underline{y}_{1}\right)$ converges to the desired Dirac delta. As pointed earlier, the above computation is invariant of the choice of $V$, so we shall choose $V$ to be the space spanned by $\left(e_{1}, \ldots, e_{k}\right)$ where $\left\{e_{i}\right\}_{i=1}^{d}$ is the canonical basis of $\mathbb{R}^{d}$. Thereafter, taking limit as $\varepsilon \rightarrow 0$, we obtain

$$
c_{1}\left(\underline{n}_{1}, V, \Lambda_{1}\right)=(2 \pi \lambda)^{-k / 2} \prod_{i=1}^{k} \frac{H_{n_{1, i}}(0)}{n_{1, i} !} .
$$

However, in order to obtain estimates for the limiting variance, we shall need bounds on $c_{1}\left(\underline{n}_{1}, V, \Lambda_{1}\right)$. Using the usual technique as sketched in [12], we obtain

$$
\begin{aligned}
\left|c_{1}\left(\underline{n}_{1}, \varepsilon, V, \Lambda_{1}\right)\right| & \leq \frac{\lambda^{-k / 2}}{\underline{n}_{1} !} \int_{\mathbb{R}^{d}} \varphi_{k}\left(\sqrt{\lambda} \varepsilon V \underline{y}_{1}\right)\left|H_{\underline{n}_{1}}\left(\underline{y}_{1}\right)\right| \varphi_{d}\left(\underline{y}_{1}\right) d \underline{y}_{1} \\
& \leq \frac{K_{1}^{d} \lambda^{-k / 2}}{\sqrt{\underline{n}_{1} !}} \int_{\mathbb{R}^{d}} \varphi_{k}\left(\sqrt{\lambda} \varepsilon V \underline{y}_{1}\right) d \underline{y}_{1}=\frac{K_{1}^{d} \lambda^{-k / 2}}{\sqrt{\underline{n}_{1} !}}
\end{aligned}
$$

where we have used the following inequality: $\sup _{x}\left|H_{l}(x) \varphi(x) / \sqrt{l !}\right| \leq K_{1}$, for some constant $K_{1}$ (see $[26]$ ).

Next, noticing that $\varphi_{k}\left(\sqrt{\lambda} \varepsilon V \underline{y}_{1}\right)$ converges to a Dirac delta on $V^{\perp}$ as $\varepsilon \rightarrow 0$, we can then write

$$
\sup _{\varepsilon} c_{1}^{2}\left(\underline{n}_{1}, \varepsilon, V, \Lambda_{1}\right) \underline{n}_{1} ! \leq K_{1}^{2 d} \lambda^{-k} .
$$




\section{Computing $c_{2}$}

The coefficient $c_{2}\left(\underline{n}_{2}, u, V, \Lambda_{2}\right)$ is the Hermite coefficient of $G_{2, u}^{V} \circ \Lambda_{2}$ (with $G_{2, u}^{V}\left(f_{2}(x)\right)$ defined in $(28)$ ), i.e.

$$
c_{2}\left(\underline{n}_{2}, u, V, \Lambda_{2}\right)=\frac{1}{\underline{n}_{2} !} \int_{\mathbb{R}^{D-d}}\left(G_{2, u}^{V} \circ \Lambda_{2}\right)\left(\underline{y}_{2}\right) H_{\underline{n}_{2}}\left(\underline{y}_{2}\right) \varphi_{D-d}\left(\underline{y}_{2}\right) d \underline{y}_{2}
$$

or, equivalently,

$$
c_{2}\left(\underline{n}_{2}, u, V, \Lambda_{2}\right)=\frac{1}{\underline{n}_{2} !} \mathbb{E}\left[\left(G_{2, u}^{V} \circ \Lambda_{2}\right)\left(\underline{Z}_{2}\right) \times H_{\underline{n}_{2}}\left(\underline{Z}_{2}\right)\right]
$$

introducing $\underline{Z}_{2}$ as a $(D-d)$-dimensional standard normal variable.

Next, using Cauchy-Schwarz inequality, we can conclude that

$c_{2}\left(\underline{n}_{2}, u, V, \Lambda_{2}\right) \leq\left\{\mathbb{E}\left[\left(G_{2, u}^{V} \circ \Lambda_{2}\right)\left(\underline{Z}_{2}\right)\right]^{2}\right\}^{1 / 2} \leq[\mathbb{P}(f(x)>u)]^{1 / 2}\left\{\mathbb{E}\left(\operatorname{det}\left(V \nabla^{2} f(x) V^{T}\right)^{4}\right\}^{1 / 4}\right.$.

Again using the invariance of $c_{2}\left(\underline{n}_{2}, u, V, \Lambda_{2}\right)$ with respect to $V$, we can choose $V$ to be the line span of $\left(e_{1}, \ldots, e_{k}\right)$, where $\left\{e_{i}\right\}_{i=1}^{d}$ is the canonical basis of $\mathbb{R}^{d}$. Writing $\left.\nabla^{2} f(x)\right|_{k \times k}$ as the top left $k \times k$ minor of $\nabla^{2} f(x)$, we have $\mathbb{E}\left(\operatorname{det}\left(V \nabla^{2} f(x) V^{T}\right)^{4}=\mathbb{E}\left(\operatorname{det}\left(\left.\nabla^{2} f(x)\right|_{k \times k}\right)^{4}\right.\right.$. Then, using Wick's formula, we can obtain an upper bound for $\mathbb{E}\left(\operatorname{det}\left(V \nabla^{2} f(x) V^{T}\right)^{4}\right.$.

On the other hand, $\mathbb{P}(f(x)>u)$ can be bounded above (and below) by the standard Mill's ratio, implying there exists $K_{2, u} \in(0, \infty)$ such that

$$
c_{2}\left(\underline{n}_{2}, u, V, \Lambda_{2}\right) \leq K_{2, u} .
$$

REMARK 3.3 Now that we have seen precise expressions for the Hermite coefficients $c_{1}\left(\underline{n}_{1}, V, \Lambda_{1}\right)$ and $c_{2}\left(\underline{n}_{2}, u, V, \Lambda_{2}\right)$, and we understand that these coefficients do not depend on the choice of $V$, we shall do away with keeping $V$ in the notation, and instead replace it by $k$, which is the dimension of $V$. In particular, we shall now redefine

$$
\begin{gathered}
c_{1}\left(\underline{n}_{1}, k, \Lambda_{1}\right) \triangleq c_{1}\left(\underline{n}_{1}, V, \Lambda_{1}\right), \\
c_{2}\left(\underline{n}_{2}, u, k, \Lambda_{2}\right) \triangleq c_{2}\left(\underline{n}_{2}, u, V, \Lambda_{2}\right),
\end{gathered}
$$

and

$$
c(\underline{n}, u, k, \Lambda) \triangleq c_{1}\left(\underline{n}_{1}, k, \Lambda_{1}\right) \times c_{2}\left(\underline{n}_{2}, u, k, \Lambda_{2}\right) .
$$

With these notations, and armed with the fact that $\mathbb{E}\left[\left(\phi_{k}\left(\partial_{k} \mathcal{T}_{V^{*}}\right)\right)^{2}\right]<\infty$, we can conclude that the following infinite expansion holds in $L^{2}$

$$
\begin{aligned}
\phi_{k}\left(\partial_{k} \mathcal{T}_{V^{*}}\right) & =\sum_{q=0}^{\infty} \sum_{\underline{n} \in \mathbb{N}^{D} ;|\underline{n}|=q} c(\underline{n}, u, k, \Lambda) \int_{\mathcal{T}_{V^{*}}} H_{\underline{n}}(Z(x)) d x \\
& \triangleq \sum_{q=0}^{\infty} J_{q}\left(\phi_{k}\left(\partial_{k} \mathcal{T}_{V^{*}}\right)\right)
\end{aligned}
$$


where $J_{q}\left(\phi_{k}\left(\partial_{k} \mathcal{T}_{V^{*}}\right)\right)$ is the projection of $\phi_{k}\left(\partial_{k} \mathcal{T}_{V^{*}}\right)$ onto the $q$-th chaos. In addition, we have the following expansion for $\mathcal{L}_{d-k, k}\left(A_{u}(f ; \mathcal{T})\right)$.

Proposition 3.1 For $f$ satisfying the assumptions set forth in Section 2.4, the following expansion holds in $L^{2}$ :

$$
\mathcal{L}_{d-k, k}\left(A_{u}(f ; \mathcal{T})\right)=\sum_{q=0}^{\infty} \sum_{\underline{n} \in \mathbb{N}^{D} ;|\underline{n}|=q} c(\underline{n}, u, k, \Lambda) \int_{\operatorname{Graff}(d, k)} \int_{\mathcal{T}_{V^{*}}} H_{\underline{n}}(Z(x)) d x d \lambda_{k}^{d}\left(V^{*}\right) .
$$

Proof: First consider the finite sum

$$
\mathcal{L}_{d-k, k}^{(Q)}\left(A_{u}(f ; \mathcal{T})\right)=\sum_{q=0}^{Q} \sum_{\underline{n} \in \mathbb{N}^{D} ;|\underline{n}|=q} c(\underline{n}, u, k, \Lambda) \int_{\operatorname{Graff}(d, k)} \int_{\mathcal{T}_{V^{*}}} H_{\underline{n}}(Z(x)) d x d \lambda_{k}^{d}\left(V^{*}\right) .
$$

Also, define $\phi_{k}^{(Q)}\left(\partial_{k} \mathcal{T}_{V^{*}}\right)$ as the projection of $\phi_{k}\left(\partial_{k} \mathcal{T}_{V^{*}}\right)$ onto the first $Q$ orders of the Hermite expansion given in (35). Then we can write

$$
\mathcal{L}_{d-k, k}^{(Q)}\left(A_{u}(f ; \mathcal{T})\right)=\int_{\operatorname{Graff}(d, k)} \phi_{k}^{(Q)}\left(\partial_{k} \mathcal{T}_{V^{*}}\right) d \lambda_{k}^{d}\left(V^{*}\right) .
$$

Writing $\|\cdot\|_{2}$ for $L^{2}$ norm, we have:

$$
\begin{aligned}
\left\|\mathcal{L}_{d-k, k}\left(A_{u}(f ; \mathcal{T})\right)-\mathcal{L}_{d-k, k}^{(Q)}\left(A_{u}(f ; \mathcal{T})\right)\right\|_{2}^{2} & =\left\|\int_{\operatorname{Graff}(d, k)}\left[\phi_{k}^{(Q)}\left(\partial_{k} \mathcal{T}_{V^{*}}\right)-\phi_{k}\left(\partial_{k} \mathcal{T}_{V^{*}}\right)\right] d \lambda_{k}^{d}\left(V^{*}\right)\right\|_{2}^{2} \\
& \leq \int_{\operatorname{Graff}(d, k)}\left\|\phi_{k}^{(Q)}\left(\partial_{k} \mathcal{T}_{V^{*}}\right)-\phi_{k}\left(\partial_{k} \mathcal{T}_{V^{*}}\right)\right\|_{2}^{2} d \lambda_{k}^{d}\left(V^{*}\right) .
\end{aligned}
$$

Next, using computations similar to those in Section 3.1, we can conclude that there exists a finite, positive $C_{\mathcal{T}}$ such that $\left\|\phi_{k}\left(\partial_{k} \mathcal{T}_{V^{*}}\right)\right\|_{2}^{2} \leq C_{\mathcal{T}}\left|\mathcal{T}_{V^{*}}\right|$. Notice also, via (35), that $\left\|\phi_{k}^{(Q)}\left(\partial_{k} \mathcal{T}_{V^{*}}\right)-\phi_{k}\left(\partial_{k} \mathcal{T}_{V^{*}}\right)\right\|_{2} \underset{Q \rightarrow \infty}{\rightarrow} 0$. We can then conclude, via the dominated convergence theorem, that

which proves Proposition 3.1.

$$
\left\|\mathcal{L}_{d-k, k}\left(A_{u}(f ; \mathcal{T})\right)-\mathcal{L}_{d-k, k}^{(Q)}\left(A_{u}(f ; \mathcal{T})\right)\right\|_{2}^{2} \underset{Q \rightarrow \infty}{\longrightarrow} 0
$$

REMARK 3.4

(i) Another convenient way of writing $\mathcal{L}_{d-k, k}\left(A_{u}(f ; \mathcal{T})\right)$ is to express the above expansion as

$$
\mathcal{L}_{d-k, k}\left(A_{u}(f ; \mathcal{T})\right)=\sum_{q=0}^{\infty} \int_{\text {Graff }(d, k)} J_{q}\left(\phi_{k}\left(\partial_{k} \mathcal{T}_{V^{*}}\right)\right) d \lambda_{k}^{d}\left(V^{*}\right) .
$$

(ii) As a consequence of [27, Lemma 3.2], we note that for any $V_{1}^{*}, V_{2}^{*} \in G r a f f(d, k)$.

$$
\mathbb{E}\left(\int_{\mathcal{T}_{V_{1}^{*}}^{*}} \int_{\mathcal{T}_{V_{2}^{*}}^{*}} H_{\underline{n}}(Z(x)) H_{\underline{m}}(Z(y)) d x d y\right)=0, \quad \text { whenever }|\underline{n}| \neq|\underline{m}|,
$$

which in turn implies that the expansion in (36) is indeed orthogonal. 


\subsection{Variance bounds}

Let us define the appropriately normalised quantities of interest

$$
\mathcal{L}_{d-k}^{\#}(\mathcal{T})=\frac{1}{|\mathcal{T}|^{1 / 2}}\left(\mathcal{L}_{d-k}\left(A_{u}(f ; \mathcal{T})\right)-\mathbb{E}\left[\mathcal{L}_{d-k}\left(A_{u}(f ; \mathcal{T})\right)\right]\right)
$$

and

$$
\mathcal{L}_{d-k, l}^{\#}(\mathcal{T})=\frac{1}{|\mathcal{T}|^{1 / 2}}\left(\mathcal{L}_{d-k, l}\left(A_{u}(f ; \mathcal{T})\right)-\mathbb{E}\left[\mathcal{L}_{d-k, l}\left(A_{u}(f ; \mathcal{T})\right)\right]\right), 1 \leq l \leq k
$$

We want to ensure that the variance of $\mathcal{L}_{d-k, k}^{\#}\left(A_{u}(f ; \mathcal{T})\right)$ converges to a finite positive quantity as $\mathcal{T} \rightarrow \mathbb{R}^{d}$, and that the variance of $\mathcal{L}_{d-k, k}^{\#}\left(A_{u}(f ; \mathcal{T})\right)$ for each $l=0, \ldots,(k-1)$ can be made as small as we wish, by choosing appropriately large set $\mathcal{T}$.

Proposition 3.2 With the above notation, the variance of $\mathcal{L}_{d-k}^{\#}$ is given by

$$
\operatorname{var}\left(\mathcal{L}_{d-k}^{\#}(\mathcal{T})\right)=\operatorname{var}\left(\mathcal{L}_{d-k, k}^{\#}(\mathcal{T})\right)+\mathrm{o}(1), \quad \text { as } \mathcal{T} \rightarrow \mathbb{R}^{d}
$$

The asymptotic variance of $\mathcal{L}_{d-k}^{\#}(\mathcal{T})$, as $\mathcal{T} \rightarrow \mathbb{R}^{d}$, is finite, non zero, and can be expressed as

$$
\lim _{\mathcal{T} \rightarrow \mathbb{R}^{d}} \operatorname{var}\left(\mathcal{L}_{d-k, k}^{\#}(\mathcal{T})\right)=\sum_{q=1}^{\infty} V_{q}^{k} \in(0, \infty)
$$

where $V_{q}^{k}=\lim _{\mathcal{T} \rightarrow \mathbb{R}^{d}} \operatorname{var}\left(\int_{\operatorname{Graff}(d, k)} J_{q}\left(\phi_{k}^{\#}\left(\partial_{k} \mathcal{T}_{V^{*}}\right)\right) d \lambda_{k}^{d}\left(V^{*}\right)\right)$, with

$\phi_{k}^{\#}\left(\partial_{k} \mathcal{T}_{V^{*}}\right)=\frac{1}{|\mathcal{T}|^{1 / 2}}\left(\phi_{k}\left(\partial_{k} \mathcal{T}_{V^{*}}\right)-\mathbb{E}\left(\phi_{k}\left(\partial_{k} \mathcal{T}_{V^{*}}\right)\right)\right)$

Using the Hermite expansion of $\mathcal{L}_{d-k, k}\left(A_{u}(f ; \mathcal{T})\right)$ and the orthogonality of the chaos expansion (see Remark $3.4($ ii $)$ ), we can formally express the variance of $\mathcal{L}_{d-k, k}\left(A_{u}(f ; \mathcal{T})\right.$ ) as

$$
\begin{aligned}
& \operatorname{var}\left(\mathcal{L}_{d-k, k}\left(A_{u}(f ; \mathcal{T})\right)\right)= \\
& \sum_{q=1}^{\infty} \operatorname{var}\left(\sum_{\underline{n} \in \mathbb{N}^{D} ;|\underline{n}|=q} c(\underline{n}, u, k, \Lambda) \int_{\operatorname{Graff}(d, k)} \int_{\mathcal{T}_{V^{*}}} H_{\underline{n}}(Z(x)) d x d \lambda_{k}^{d}\left(V^{*}\right)\right)= \\
& \sum_{q=1}^{\infty} \sum_{\substack{|\underline{n}|=|\underline{m}|=q \\
\underline{n}, \underline{m} \in \mathbb{N}^{D}}} c(\underline{n}, u, k, \Lambda) c(\underline{m}, u, k, \Lambda) \iint_{U^{*}, V^{*} \in G r(d, k)}\left\{\int_{\mathcal{T}_{U^{*}}} \int_{\mathcal{T}_{V^{*}}} \mathbb{E}\left[H_{\underline{n}}(Z(x)) H_{\underline{m}}(Z(y))\right] d x d y\right\} d \lambda_{k}^{d}\left(U^{*}\right) d \lambda_{k}^{d}\left(V^{*}\right) .
\end{aligned}
$$

The sketch and main arguments (e.g. Arcones bound) to prove Proposition 3.2 are given in [14], with an extra step for the term $o(1)$ which follows from [6]. The main difficulty relies 
then, once again, in the fact that we do not integrate simply on a $d$-dimensional box, but on Grassmanians, which requires tricks to circumvent the difficulty of computations.

Proof of Proposition 3.2. $\quad$ First let us show that $\operatorname{var}\left(\mathcal{L}_{d-k, k}^{\#}(\mathcal{T})\right)<\infty$. We have, using (19), then (35),

$$
\begin{aligned}
& \operatorname{var}\left(\mathcal{L}_{d-k, k}^{\#}(\mathcal{T})\right)= \\
& \frac{1}{|\mathcal{T}|} \int_{\operatorname{Graff}(d, k)} \int_{\operatorname{Graff}(d, k)} \operatorname{cov}\left(\phi_{k}\left(\partial_{k} \mathcal{T}_{V^{*}}\right), \phi_{k}\left(\partial_{k} \mathcal{T}_{U^{*}}\right)\right) d \lambda_{k}^{d}\left(V^{*}\right) d \lambda_{k}^{d}\left(U^{*}\right) \\
= & \frac{1}{|\mathcal{T}|} \sum_{q=1}^{\infty} \sum_{|\underline{n}|=q} \sum_{|\underline{m}|=q} c(\underline{n}, u, k, \Lambda) c(\underline{m}, u, k, \Lambda) A(\underline{n}, \underline{m}, u, k, \mathcal{T})
\end{aligned}
$$

with

$$
\begin{aligned}
& A(\underline{n}, \underline{m}, u, k, \mathcal{T}):= \\
& \quad \int_{\operatorname{Graff}(d, k) \operatorname{Graff}(d, k)}\left[\int_{x \in \partial_{k}} \int_{\mathcal{T}^{*}} \int_{y \in \partial_{k}} \mathbb{\mathcal { T } _ { V ^ { * } }}\left[H_{\underline{n}}(Z(x)) H_{\underline{m}}(Z(y))\right] \mathcal{H}_{k}(d x) \mathcal{H}_{k}(d y)\right] d \lambda_{k}^{d}\left(U^{*}\right) d \lambda_{k}^{d}\left(V^{*}\right) .
\end{aligned}
$$

Notice that since $Z(x)$ and $Z(y)$, individually, are standard Gaussian vectors, then using Mehler's formula (or equivalently, the diagram formula), we have that for $|\underline{n}|=|\underline{m}|=q$,

$$
\begin{aligned}
& A(\underline{n}, \underline{m}, u, k, \mathcal{T})=\sum_{\substack{d_{i j} \geq 0 \\
\sum_{i} d_{i j}=n_{j} \\
\sum_{j} d_{i j}=m_{i}}} \underline{n} ! \underline{m} ! \\
& \times \int_{U^{*} \in \operatorname{Graff}(d, k)} \int_{x \in \partial_{k}}\left[\int_{\mathcal{T}_{U^{*}}}\left[\int_{V^{*} \in \operatorname{Graff}(d, k) w \in \partial_{k}\left(x-\mathcal{T}_{\left.V^{*}\right)}\right.} \prod_{1 \leq i, j \leq D} \frac{\gamma_{i j}^{d_{i j}}(w)}{d_{i j} !} \mathcal{H}_{k}(d w) d \lambda_{k}^{d}\left(V^{*}\right)\right] \mathcal{H}_{k}(d x) d \lambda_{k}^{d}\left(U^{*}\right)\right. \\
& \leq \sum_{\substack{d_{i j} \geq 0 \\
\sum_{i j} d_{i j}=n_{j} \\
\sum_{j} d_{i j}=m_{i}}} \underline{n} \underline{m} !\left[\int_{V^{*} \in \operatorname{Graff}(d, k) w \in \partial_{k}\left(2 \mathcal{T}_{\left.V^{*}\right)}\right.} \prod_{1 \leq i, j \leq D} \frac{\left|\gamma_{i j}^{d_{i j}}(w)\right|}{d_{i j} !} \mathcal{H}_{k}(d w) d \lambda_{k}^{d}\left(V^{*}\right)\right] \int_{U^{*} \in \operatorname{Graff}(d, k) x \in \partial_{k}\left(\mathcal{T}_{U^{*}}\right)} \mathcal{H}_{k}(d x) d \lambda_{k}^{d}\left(U^{*}\right)
\end{aligned}
$$

where the inequality comes from the result of the observation that $\left(x-\mathcal{T}_{V^{*}}\right)=(x-\mathcal{T})_{V^{*}}$. Before proceeding any further, we may observe the following.

Lemma 3.1 Let $\theta$ be a nonnegative real valued, integrable function defined on $\mathbb{R}^{d}$, then

$$
\int_{V^{*} \in \operatorname{Graff}(d, k)} \int_{w \in \partial_{k}\left(2 \mathcal{T}_{V}^{*}\right)} \theta(w) \mathcal{H}_{k}(d w) d \lambda_{k}^{d}\left(V^{*}\right) \leq\left[\begin{array}{l}
d \\
k
\end{array}\right] \int_{\mathbb{R}^{d}} \theta(z) d z .
$$


Proof: The double integral in question can be upper bounded by first replacing the integral over $\partial_{k}\left(2 \mathcal{T}_{V^{*}}\right)$ by integral over $V^{*}$. Then, since $\operatorname{Graff}(d, k)$ is isometric to $\operatorname{Gr}(d, k) \times \mathbb{R}^{d-k}$, we note that, for any fixed $V \in \operatorname{Gr}(d, k)$, we have $\cup_{x \in \mathbb{R}^{d-k}}(V+x)=\mathbb{R}^{d}$. Therefore the above integral can be bounded above by

$$
\int_{V \in \operatorname{Gr}(d, k)}\left(\int_{\mathbb{R}^{d}} \theta(w) d w\right) d \sigma_{k}^{d}(V)
$$

where $\sigma_{k}^{d}$ is the invariant measure on the Grassmannian $\operatorname{Gr}(d, k)$ such that

$$
\sigma_{k}^{d}(\operatorname{Gr}(d, k))=\left[\begin{array}{l}
d \\
k
\end{array}\right]
$$

which proves the assertion of the lemma.

In view of Lemma 3.1, an upper bound for $A(\underline{n}, \underline{m}, u, k, \mathcal{T})$ can be obtained as

$$
\begin{aligned}
& A(\underline{n}, \underline{m}, u, k, \mathcal{T}) \\
& \leq\left[\begin{array}{l}
d \\
k
\end{array}\right]_{\substack{d_{i j} \geq 0 \\
\sum_{i} d_{i j}=n_{j} \\
\sum_{j} d_{i j}=m_{i}}}^{2} \underline{n} ! \underline{m} !\left[\int_{w \in \mathbb{R}^{d}} \prod_{1 \leq i, j \leq D} \frac{\left|\gamma_{i j}^{d_{i j}}(w)\right|}{d_{i j} !} d w\right] \int_{\substack{x \in \mathcal{T} \\
\mathcal{H}_{d}(d x)}} \mathcal{H}
\end{aligned}
$$

where in the second integral we have used the Crofton formula (8).

Further, under hypothesis (H2), and for $|n|=|m|=q$, there exists a constant $C^{*}$ such that

$$
\sum_{\substack{d_{i j} \geq 0 \\ \sum_{i} d_{i j}=n_{j} \\ \sum_{j} d_{i j}=m_{i}}} \underline{n} ! \underline{m} ! \prod_{1 \leq i, j \leq D} \frac{\left|\gamma_{i j}^{d_{i j}}(w)\right|}{d_{i j} !} \leq C^{*} \psi^{q}(w) .
$$

Therefore we obtain that, for $|n|=|m|=q$,

$$
A(\underline{n}, \underline{m}, u, k, \mathcal{T}) \leq C^{*}|\mathcal{T}|\left[\begin{array}{l}
d \\
k
\end{array}\right]^{2} \int_{\mathbb{R}^{d}} \psi^{q}(w) d w .
$$

Next, we prove that $|\mathcal{T}|^{-1} A(\underline{n}, \underline{m}, u, k, \mathcal{T})$ converges as $\mathcal{T} \rightarrow \mathbb{R}^{d}$, for which we shall check it is Cauchy in $\mathcal{T}$. For $r>0$, writing $(1+r) \mathcal{T}$ to denote the inflated set $\{x(1+r): x \in \mathcal{T}\}$, we have

$$
\begin{aligned}
& \left.||(1+r) \mathcal{T}\right|^{-1} A(\underline{n}, \underline{m}, u, k,(1+r) \mathcal{T})-|\mathcal{T}|^{-1} A(\underline{n}, \underline{m}, u, k, \mathcal{T}) \mid \leq \\
& \frac{1}{|(1+r) \mathcal{T}|}|A(\underline{n}, \underline{m}, u, k,(1+r) \mathcal{T})-A(\underline{n}, \underline{m}, u, k, \mathcal{T})|+\frac{\left.||(1+r) \mathcal{T}\right|^{-1}|\mathcal{T}|-1 \mid}{|\mathcal{T}|} A(\underline{n}, \underline{m}, u, k, \mathcal{T}) \\
& :=I+I I
\end{aligned}
$$


Clearly, $I I$ converges to zero as $\mathcal{T}$ and $r$ increase to infinity. For part $I$, notice that the difference $|A(\underline{n}, \underline{m}, u, k,(1+r) \mathcal{T})-A(\underline{n}, \underline{m}, u, k, \mathcal{T})|$ can be shown to be of the same order as

$$
|(1+r) \mathcal{T} \backslash \mathcal{T}| \int_{(1+r) \mathcal{T} \backslash \mathcal{T}} \psi^{q}(w) d w .
$$

The coefficient of the integral above, when compared with $|(1+r) \mathcal{T}|^{-1}$, converges to one. However, since the domain of the integral escapes to infinity, therefore the integral converges to zero.

Hence, we can conclude that the sequence $|\mathcal{T}|^{-1} A(\underline{n}, \underline{m}, u, k, \mathcal{T})$ is Cauchy in the variable $\mathcal{T}$, meaning that, for $|\underline{n}|=|\underline{m}|$,

$$
|\mathcal{T}|^{-1} A(\underline{n}, \underline{m}, u, k, \mathcal{T}) \rightarrow A(\underline{n}, \underline{m}, u, k) \quad \text { as } \mathcal{T} \rightarrow \mathbb{R}^{d} \quad \text { (or, equivalently, as } T \rightarrow \infty \text { ) }
$$

where the limit $A(\underline{n}, \underline{m}, u, k)$, using the arguments of Lemma 3.1, can be expressed as

$$
A(\underline{n}, \underline{m}, u, k)=\left[\begin{array}{c}
d \\
k
\end{array}\right]^{2} \underline{n} \underline{\underline{m} !} \sum_{\substack{d_{i j} \geq 0 \\
\sum_{i} d_{i j}=n_{j} \\
\sum_{j} d_{i j}=m_{i}}} \int_{\mathbb{R}^{d}} \prod_{1 \leq i, j \leq D} \frac{\gamma_{i j}^{d_{i j}}(w)}{d_{i j} !} \mathcal{H}_{d}(d w),
$$

which, in turn implies that, $\operatorname{var}\left(\int_{\operatorname{Graff}(d, k)} J_{q}\left(\phi_{k}^{\#}\left(\partial_{k} \mathcal{T}_{V^{*}}\right)\right) d \lambda_{k}^{d}\left(V^{*}\right)\right) \rightarrow V_{q}^{k}$, as $\mathcal{T} \rightarrow \mathbb{R}^{d}$.

\section{FinitenESS OF THE LIMITING VARIANCE}

We shall proceed as usual (see [12] or [14]). Introducing $\Pi_{Q}\left(\mathcal{L}_{d, d-k}^{\#}\left(A_{u}(f ; \mathcal{T})\right)\right)$ as the projection of $\mathcal{L}_{d, d-k}^{\#}\left(A_{u}(f ; \mathcal{T})\right)$ onto the first $Q$ chaos, we shall show that

$$
\operatorname{var}\left(\mathcal{L}_{d, d-k}^{\#}\left(A_{u}(f ; \mathcal{T})\right)-\Pi_{Q}\left(\mathcal{L}_{d, d-k}^{\#}\left(A_{u}(f ; \mathcal{T})\right)\right)\right) \underset{Q \rightarrow \infty}{\longrightarrow} 0, \text { uniformly in } \mathcal{T}
$$

and conclude the finiteness of the limiting variance by a simple application of Fatou's lemma.

Let us begin with observing that $\mathcal{L}_{d, d-k}\left(A_{u}(f ; \mathcal{T})\right)$ is an additive set functional. In particular, the set $\mathcal{T}$ can be written, as in [6] as a union of disjoint unit cuboids (w.l.o.g. let $T$ be integer). Therefore, $\mathcal{L}_{d, d-k}\left(A_{u}(f ; \mathcal{T})\right)$ can be written as a sum of a stationary sequence of random variables where these random variables are an evaluation of $\mathcal{L}_{d, d-k}\left(A_{u}(f ; \cdot)\right.$ on $[0,1)^{d}$, and its various integer shifts.

Next invoking stationarity of the field $F$ (and also of $Z$ ), we know that the variance of the sum of a stationary sequence is of the order of the cardinality of the sum if the covariance decays at an appropriate rate. Using this precise argument, and following the computations of [6], we can conclude (44). In following the arguments of [6], it is important to note that our estimates for the coefficients in the Hermite expansion match with those in [6].

Now we shall show that the variance corresponding to lower dimensional faces of $\mathcal{T}_{V^{*}}$, is indeed $o(1)$ for large $\mathcal{T}$ as expressed in Proposition 3.2. 
Recall the decomposition of $\mathcal{L}_{d-k, k}$ from equation (18). Then,

$\operatorname{var}\left(\mathcal{L}_{d-k}\left(A_{u}(f ; \mathcal{T})\right)\right)=\sum_{l=0}^{k} \operatorname{var}\left(\mathcal{L}_{d-k, l}\left(A_{u}(f ; \mathcal{T})\right)\right)+2 \sum_{l<m} \operatorname{cov}\left(\mathcal{L}_{d-k, l}\left(A_{u}(f ; \mathcal{T})\right), \mathcal{L}_{d-k, m}\left(A_{u}(f ; \mathcal{T})\right)\right)$

It suffices to show that $\operatorname{var}\left(\mathcal{L}_{d-k, l}\left(A_{u}(f ; \mathcal{T})\right)\right)=o(|\mathcal{T}|)$ for each $l=0, \ldots,(k-1)$, in order to conclude the second part of the assertion in equation (40).

Let us define

$$
R(d, k, l, \mathcal{T})=\frac{1}{|\mathcal{T}|^{1 / 2}} \int_{\operatorname{Graff}(d, k)}\left|\partial_{l} \mathcal{T}_{V^{*}}\right|^{1 / 2} \phi_{l}^{\#}\left(\mathcal{T}_{V^{*}}\right) d \lambda_{k}^{d}\left(V^{*}\right) .
$$

In view of $\partial_{l} \mathcal{T}=\bigcup_{V^{*} \in \operatorname{Graff}(d, k)} \partial_{l} \mathcal{T}_{V^{*}}$, and the above computations leading to $A(\underline{n}, \underline{m}, u, k)$, we note that $\operatorname{var}(R(d, k, l, \mathcal{T}))$ can be shown to be $O\left(\left|\partial_{l} \mathcal{T}\right|\right)$, or equivalently $O\left(\mathcal{T}^{l}\right)$ under the assumption (H2), implying that the lower dimensional faces, asymptotically, do not contribute to the variance of $\mathcal{L}_{d-k}^{\#}\left(A_{u}(f ; \mathcal{T})\right)$.

\section{NONDEGENERACY OF THE LIMIT}

Finally, it remains to show that $\lim _{\mathcal{T} \rightarrow \mathbb{R}^{d}} \operatorname{var}\left(\mathcal{L}_{d-k, k}^{\#}\left(A_{u}(f ; \mathcal{T})\right)\right)>0$. Using the orthogonality of chaos, it suffices to show that $V_{1}^{k}>0$.

First, we shall simplify the expression for $V_{1}^{k}(\mathcal{T})$ by introducing the canonical basis $\left(\underline{e}_{i}\right)_{1 \leq i \leq D}$ of $\mathbb{R}^{D}$ in (42), and writing

$$
V_{1}^{k}(\mathcal{T})=\frac{1}{|\mathcal{T}|} \sum_{i=1}^{D} \sum_{j=1}^{D} c\left(\underline{e}_{i}, u, k, \Lambda\right) c\left(\underline{e}_{j}, u, k, \Lambda\right) A\left(\underline{e}_{i}, \underline{e}_{j}, u, k, \mathcal{T}\right) .
$$

Then, writing $\left\{\underline{e}_{i 1}\right\}_{i=1}^{d},\left\{\underline{e}_{j 2}\right\}_{j=1}^{D-d}$ for canonical basis of dimension $d,(D-d)$ respectively, and observing that $c_{1}\left(\underline{e}_{i 1}, k, \Lambda_{1}\right)=0$ (by (30)), the limiting variance corresponding to the first chaos, again using equation (30) for precise expression of $c_{1}\left(\underline{0}, k, \Lambda_{1}\right)$, is given by

$$
V_{1}^{k}=(2 \pi \lambda)^{-k} \sum_{i=d+1}^{D} \sum_{j=d+1}^{D} c_{2}\left(\underline{e}_{i 2}, u, k, \Lambda\right) c_{2}\left(\underline{e}_{j 2}, u, k, \Lambda\right) A\left(\underline{e}_{i 2}, \underline{e}_{j 2}, u, k\right)
$$

with $A\left(\underline{e}_{i 2}, \underline{e}_{j 2}, u, k\right)$ as defined in (43), given by

$$
A\left(\underline{e}_{i 2}, \underline{e}_{j 2}, u, k\right)=\left[\begin{array}{c}
d \\
k
\end{array}\right]^{2} \int_{\mathbb{R}^{d}} \gamma_{\underline{e}_{i 2}, e_{j 2}}(w) \mathcal{H}_{k}(d w)
$$

where $\gamma_{\underline{e}_{i},}, \underline{e}_{j 2}$ denotes the covariance function corresponding to the pair of indices which correspond to the position of 1 's in $\left(\underline{0}, \underline{e}_{i 2}\right)$ and $\left(\underline{0}, \underline{e}_{j 2}\right)$, respectively, where $\underline{0}$ is a $d$-dimensional row vector of zeros.

As in [6], we have that $A\left(\underline{e}_{i 2}, \underline{e}_{j 2}, u, k\right)=0$, whenever $(i, j) \neq(D, D)$, thus further simplifying (46) to

$$
V_{1}^{k}=c_{2}^{2}\left(\underline{e}_{D 2}, u, k, \Lambda_{2}\right) A\left(\underline{e}_{D 2}, \underline{e}_{D 2}, u, k\right) .
$$


We shall estimate separately the two terms appearing above.

Let us begin with $c_{2}\left(\underline{e}_{D 2}, u, k, \Lambda_{2}\right)$, for which we recall $(32)$

$$
\begin{aligned}
c_{2}\left(\underline{e}_{D 2}, u, k, \Lambda_{2}\right) & =\int_{\mathbb{R}^{D-d}}\left(G_{2, u}^{V} \circ \Lambda_{2}\right)\left(\underline{y}_{2}\right) H_{\underline{e}_{D 2}}\left(\underline{y}_{2}\right) \varphi_{D-d}\left(\underline{y}_{2}\right) d \underline{y}_{2} \\
& =\int_{\mathbb{R}^{D-d-1}} \varphi_{D-d-1}\left(\underline{y}_{2}^{*}\right)\left(\int_{\mathbb{R}}\left(G_{2, u}^{V} \circ \Lambda_{2}\right)\left(\underline{y}_{2}\right) y_{2 D} \varphi\left(y_{2 D}\right) d y_{2 D}\right) d \underline{y}_{2}^{*} .
\end{aligned}
$$

Let us consider the lower triangular matrix $\Lambda_{2}$ such that its first element $\left(\Lambda_{2}\right)_{11}$ is 1 (as in $[6])$, i.e. of the form $\Lambda_{2}=\left(\begin{array}{cc}L & 0 \\ \underline{\gamma}^{T} & l\end{array}\right)$, with $U$ a lower triangular $(D-d-1) \times(D-d-1)$ matrix, $\underline{\gamma}^{T}$ a $1 \times(D-d-1)$ matrix, and $l>0$. With the above notation, we can write

$$
G_{2, u}^{V} \circ \Lambda_{2}\left(\underline{y}_{2}\right)=\operatorname{det}\left(V \mathcal{M}\left(L \underline{y}_{2}^{*}\right) V^{T}\right) 1_{\left\{\underline{\gamma}_{2}^{*}+l y_{2 D} \geq u\right\}}
$$

where $\mathcal{M}\left(L \underline{y}_{2}^{*}\right)$ is the symmetric matrix obtained by appropriately arranging the elements of the vector $L \underline{y}_{2}^{*}$.

We can certainly think of the map $\underline{y}_{2}^{*} \mapsto \mathcal{M}\left(L \underline{y}_{2}^{*}\right)$ as a linear map, therefore, there exists $a_{i j}^{b}$ such that

$$
\mathcal{M}\left(L \underline{y}_{2}^{*}\right)_{i j}=\sum_{b=1}^{D-d-1} a_{i j}^{b} \underline{y}_{2, b}^{*} .
$$

Again recalling that $c_{2}$ does not depend on the choice of $V$, we shall fix the matrix $V$ as $\left[I_{k} ; \underline{0}\right]$, where $I_{k}$ is $k \times k$ identity matrix and $\underline{0}$ is a $k \times(d-k)$ matrix of zeros. Then,

$$
\left(V \mathcal{M}\left(L \underline{y}_{2}^{*}\right) V^{T}\right)=\left.\mathcal{M}\left(L \underline{y}_{2}^{*}\right)\right|_{k \times k}
$$

where the right side is the notation for the top left $k \times k$ minor of $\mathcal{M}\left(L \underline{y}_{2}^{*}\right)$.

This latter argument is key, since the next computations will then be similar as those done in a $d$-dimensional box $([6])$. We now give brief sketch of major steps involved to provide an overview of the full computation.

$$
\operatorname{det}\left(\left.\mathcal{M}\left(L \underline{y}_{2}^{*}\right)\right|_{k \times k}\right)=\sum_{\sigma \in \mathcal{S}_{k}} \operatorname{sgn}(\sigma) \prod_{i=1}^{k}\left[\sum_{b=1}^{D-d-1} a_{i \sigma(i)}^{b} \underline{y}_{2, b}^{*}\right]
$$

Subsequently, using arguments similar to those in Lemma A.2 of [6] together with isotropy, we can obtain a Hermite expansion for the determinant as follows

$$
\operatorname{det}\left(V \mathcal{M}\left(L \underline{y}_{2}^{*}\right) V^{T}\right)=\sum_{\underline{n} \in \mathbb{N}^{D-d-1}:|\underline{n}|=k} \alpha_{\underline{n}}(L, V) H_{\underline{n}}\left(\underline{y}_{2}^{*}\right) .
$$

Combining (48), (49) and (50), and using that $y \varphi(y)=-\varphi^{\prime}(y)$ to compute the integrand on $y_{2 D}$, we obtain the following (for more details, we refer the reader to the proof of Lemma 
2.2 of $[6])$

$$
\begin{aligned}
& c_{2}\left(\underline{e}_{D 2}, u, k, \Lambda_{2}\right)=\sum_{\underline{n} \in \mathbb{N}^{D-d-1}:|\underline{n}|=k} \alpha_{\underline{n}}(L, V) \int_{\mathbb{R}^{D-d-1}} H_{\underline{n}}\left(\underline{y}_{2}^{*}\right) \varphi\left(\frac{1}{l}\left(u-\left\langle\underline{\gamma}, \underline{y}_{2}^{*}\right\rangle\right)\right) \varphi_{D-d-1}\left(\underline{y}_{2}^{*}\right) d \underline{y}_{2}^{*} \\
& =\sum_{\underline{n} \in \mathbb{N}^{D-d-1}:|\underline{n}|=k} \alpha_{\underline{n}}(L, V)(-1)^{k} \int_{\mathbb{R}^{D-d-1}} \varphi\left(\frac{1}{l}\left(u-\left\langle\underline{\gamma}, \underline{y}_{2}^{*}\right\rangle\right)\right) \varphi_{D-d-1}^{(n)}\left(\underline{y}_{2}^{*}\right) d \underline{y}_{2}^{*} \\
& =l H_{k}(u) \varphi(u) \sum_{\underline{n} \in \mathbb{N}^{D-d-1}:|\underline{n}|=k} \alpha_{\underline{n}}(L, V) H_{\underline{n}}(\underline{\gamma}) \\
& =l H_{k}(u) \varphi(u) \operatorname{det}\left(V \mathcal{M}(L \underline{\gamma}) V^{T}\right) \text {. }
\end{aligned}
$$

Since we can write $\mathcal{M}(L \underline{\gamma})=-\lambda I_{k}$ with $\lambda=-r_{i i}(0)$, then $c_{2}\left(\underline{e}_{D 2}, u, k, \Lambda_{2}\right)=l H_{k}(u) \varphi(u)(-\lambda)^{k}$, i.e.

$$
c_{2}\left(\underline{e}_{D 2}, u, k, \Lambda_{2}\right)=l H_{k}(u) \varphi(u)(-\lambda)^{k}
$$

by way of choosing $V=\left[I_{k} ; \underline{0}\right]$.

Moreover, as in [6], we can write

$$
\int_{w \in \mathbb{R}^{D}} \gamma_{\underline{e}_{D 2}, \underline{e}_{D 2}}(w) \mathcal{H}_{k}(d w)=(2 \pi)^{d} h(0) l^{-2}
$$

where we recall that $h(0)$ is the spectral density of the field $f$ evaluated at 0 .

Finally, putting together the estimates obtained in (51) and (52) in the following

$$
V_{1}^{k}=(2 \pi)^{-k} l^{2} H_{k}^{2}(u) \varphi^{2}(u) \lambda^{k}\left[\begin{array}{l}
d \\
k
\end{array}\right]^{2} \int_{w \in \mathbb{R}^{D}} \gamma_{\underline{e}_{D 2}, \underline{e}_{D 2}}(w) \mathcal{H}_{k}(d w),
$$

we obtain

$$
V_{1}^{k}=(2 \pi)^{d-k} H_{k}^{2}(u) \varphi^{2}(u) \lambda^{k} h(0)\left[\begin{array}{l}
d \\
k
\end{array}\right]^{2}
$$

from which we deduce that $V_{1}^{k}>0$, hence the second part of Proposition 3.2.

\subsection{Extension of Breuer-Major theorem to affine Grassmannian case}

Here we just give a sketchy recall of the literature on CLTs of Breuer-Major type, that can be found in $[19,20]$.

In 1983, Breuer-Major provided a CLT for a 1-dimensional centered stationary Gaussian sequence satisfying some condition on its correlation function. This result was first extended 
by Giraitis and Surgailis ([8]) when considering a continuous time setting, then by Arcones ([2]) with a powerful result holding for general $d$. The proof, in the discrete case, is based on the method of cumulants and diagram formulae. Estrade and Léon rewrote it explicitly (see [6], Proposition 2.4) in the continuous case following the Nourdin et al's proof ([20]) based on Malliavin calculus. To avoid mimicking the proof a second time, we shall point out the main quantities that deserve some care, due to our general setting. Let us first state Breuer-Major theorem in this setting.

Proposition 3.3 Let $\mathcal{T}$ be a d-dimensional box $[-T, T]^{d}$, and let $f$ be a mean zero, unit variance, isotropic Gaussian random field defined on $\mathbb{R}^{d}$ with $C^{3}$ trajectories. Under the assumptions (H1) to (H3), for any positive integer $Q$, the projection onto the first $Q$ chaos $\Pi^{Q}\left(\mathcal{L}_{d-k, k}^{\#}\left(A_{u}(f ; \mathcal{T})\right)\right)$ satisfies

$$
\Pi^{Q}\left(\mathcal{L}_{d-k, k}^{\#}\left(A_{u}(f ; \mathcal{T})\right)\right) \stackrel{d}{\longrightarrow} \mathcal{N}\left(0, \sum_{q=1}^{Q} V_{q}^{k}\right) \quad \text { as } \mathcal{T} \rightarrow \mathbb{R}^{d}
$$

where $V_{q}^{k}$ is defined in Proposition 3.2.

Indeed, we have

$$
\begin{aligned}
& \frac{1}{|\mathcal{T}|^{1 / 2}} \int_{\operatorname{Graff}(d, k)} \phi_{k}\left(\partial_{k} \mathcal{T}_{V^{*}}\right) d \lambda_{k}^{d}\left(V^{*}\right)=\frac{1}{|\mathcal{T}|^{1 / 2}} \int_{\operatorname{Graff}(d, k) \partial_{k} \mathcal{T}_{V}} G^{V}\left(f_{1}, f_{2}\right)(x) d x d \lambda_{k}^{d}\left(V^{*}\right) \\
& =\frac{1}{\left|\int_{\operatorname{Graff}(d, k)} \int_{\partial_{k}} d x d \lambda_{k}^{d}\left(V^{*}\right)\right|^{1 / 2}} \int_{\operatorname{Graff}(d, k)} \int_{\partial_{k}} G_{\mathcal{T}_{V^{*}}} G^{V}\left(f_{1}, f_{2}\right)(x) d x d \lambda_{k}^{d}\left(V^{*}\right) .
\end{aligned}
$$

where $f_{1}=\nabla f, f_{2}=\left(\nabla^{2} f, f\right)$, and $G^{V}\left(f_{1}, f_{2}\right)=G_{1}^{V}\left(f_{1}\right) \times G_{2, u}^{V}\left(f_{2}\right)$ as defined in $(27)$ and (28).

Considering the projection onto the first $Q$ chaos, $\Pi^{Q}\left(\mathcal{L}_{d-k, k}^{\#}\left(A_{u}(f ; \mathcal{T})\right)\right)$, defined in $(44)$, we can write, as in the proof of Theorem 2.2 in [20] (or in [6]),

$$
\Pi^{Q}\left(\mathcal{L}_{d-k, k}^{\#}(\mathcal{T})\right)=\sum_{q=1}^{Q} I_{q}\left(g_{k, q}^{\mathcal{T}}\right)
$$

where $I_{q}(f)$ denotes the multiple Wiener-Itô integral (of order q) of $f$ with respect to $W$, and

$g_{k, q}^{\mathcal{T}}:=\frac{b_{\underline{m}}^{k}}{\left|\int_{\operatorname{Graff}(d, k) \partial_{k} T_{V^{*}}} d x d \lambda_{k}^{d}\left(V^{*}\right)\right|^{1 / 2}} \int_{\operatorname{Graff}(d, k)} \int_{\partial_{k} \mathcal{T}_{V^{*}}} \sum_{\underline{m} \in\{1,2, \cdots, D\}^{q}} u_{x, m_{1}} \otimes \cdots \otimes u_{x, m_{q}} d x d \lambda_{k}^{d}\left(V^{*}\right)$

where $b_{\underline{m}}^{k}$ are such that the mapping $\underline{m} \rightarrow b_{\underline{m}}^{k}$ is symmetric on $\{1, \cdots, D\}^{q}$, and we have again used isotropy to observe that $b_{\underline{m}}^{k}$ depends on $V^{*}$ only through its dimension, which 
is $k$. Moreover, the functions $\left(u_{x, j}\right)_{1 \leq j \leq D}$ are orthogonal in $L^{2}\left(\mathbb{R}^{d}\right)$ such that for the field $Z(x)$ defined in (15),

$$
Z_{j}(x)=\int_{\mathbb{R}^{d}} u_{x, j}(w) d W(w)
$$

where $W$ is the complex Brownian measure on $\mathbb{R}^{d}$.

Note that, in writing (53), we have used the Fubini theorem to interchange the Wiener-Itô integral and the integral over the space $\underset{V^{*} \in \operatorname{Graff}(d, k)}{\cup} \partial_{k} \mathcal{T}_{V^{*}}$.

In order to prove the CLT of $\Pi^{Q}\left(\mathcal{L}_{d-k, k}^{\#}\left(A_{u}(f ; \mathcal{T})\right)\right)$, it is enough to check that, for $1 \leq$ $p, q \leq Q$, (see [19] or [20], and for the notation, [6])

$$
\left\|\delta_{p q} V_{p}^{k}-\frac{1}{q}\left\langle\mathrm{D} I_{p}\left(g_{k, p}^{\mathcal{T}}\right), \mathrm{D} I_{q}\left(g_{k, q}^{\mathcal{T}}\right)\right\rangle_{\mathcal{H}}\right\|_{2} \rightarrow 0 \text { as } \mathcal{T} \rightarrow \mathbb{R}^{d}
$$

where $V_{q}^{k}$ is defined in Proposition 3.2, and D denotes the Malliavin derivative. Standard analysis as in [20] can be invoked to conclude that it suffices to check that, for $p \leq q$,

$$
\left\|\frac{1}{q}\left\langle\mathrm{D} I_{p}\left(g_{k, p}^{\mathcal{T}}\right), \mathrm{D} I_{q}\left(g_{k, q}^{\mathcal{T}}\right)\right\rangle_{\mathcal{H}}\right\|_{2} \rightarrow 0 \text { as } \mathcal{T} \rightarrow \mathbb{R}^{d}
$$

which holds since, on one hand, for the case $p=q$ we have $\left\|g_{k, q}^{\mathcal{T}}\right\|_{\mathcal{H}^{q}}^{2}=V_{q}^{k}(\mathcal{T})$ which is shown to converge to $V_{q}^{k}$ in Proposition 3.2. On the other hand, the $e$-th contraction of $g_{k, p}^{\mathcal{T}}$ satisfies, for $e<p$,

$$
\left\|g_{k, p}^{\mathcal{T}} \underset{e}{\otimes} g_{k, p}^{\mathcal{T}}\right\|_{\mathcal{H}^{2(p-e)}}^{2} \leq\left(C^{p} \sum_{\underline{m} \in\{1,2, \cdots, D\}^{p}}\left|b_{\underline{m}}\right|^{2}\right)^{2} \Psi(k)
$$

with some constant $C$, and under (H2),

$$
\begin{aligned}
& \Psi(k):= \\
& \int_{(\operatorname{Graff}(d, k))^{4}} \int_{\partial_{k}} \ldots \int_{\mathcal{T}_{1}^{*} \partial_{k} \mathcal{T}_{V_{4}^{*}}^{*}} \frac{\psi^{e}\left(t_{1}-t_{2}\right) \psi^{e}\left(t_{3}-t_{4}\right) \psi^{p-e}\left(t_{1}-t_{3}\right) \psi^{p-e}\left(t_{2}-t_{4}\right)}{\left|\int_{\operatorname{Graff}(d, k) \partial_{k} \mathcal{T}_{V^{*}}} d x d \lambda_{k}^{d}\left(V^{*}\right)\right|^{2}} \prod_{i=1}^{4} d t_{i} d \lambda_{k}^{d}\left(V_{i}^{*}\right) .
\end{aligned}
$$

Like in [6], we note that $\psi^{e}\left(t_{3}-t_{4}\right) \psi^{p-e}\left(t_{1}-t_{3}\right) \leq \psi^{p}\left(t_{3}-t_{4}\right)+\psi^{p}\left(t_{1}-t_{3}\right)$, and by Lemma 3.1, we have

$$
\int_{\operatorname{Graff}(d, k)} \int_{\partial_{k}} \psi_{\mathcal{T}^{*}} \psi^{p}\left(t_{1}-t_{3}\right) d s_{3}<\left[\begin{array}{l}
d \\
k
\end{array}\right] \int_{\mathbb{R}^{d}} \psi(z) d z<\infty
$$

which matches the estimates of [6], and thus we can follow the rest of the arguments verbatim to conclude that for some finite, combinatorial constant $C(k)$, we have

$$
\Psi(k) \leq C(k) \frac{\left|\int_{\operatorname{Graff}(d, k) \partial_{k}} \int_{\mathcal{T}_{V^{*}}} d x d \lambda_{k}^{d}\left(V^{*}\right)\right|}{\left|\int_{\operatorname{Graff}(d, k) \partial_{k}} \int_{\mathcal{T}_{V^{*}}} d x d \lambda_{k}^{d}\left(V^{*}\right)\right|^{2}}=\frac{C(k)}{\left|\int_{\operatorname{Graff}(d, k) \partial_{k} \mathcal{T}_{V^{*}}} d x d \lambda_{k}^{d}\left(V^{*}\right)\right|} \rightarrow 0 \quad \text { as } \mathcal{T} \rightarrow \mathbb{R}^{d} .
$$


This concludes the proof of Proposition 3.3.

Collating Propositions 3.2 and 3.3 leads to the main result, that is the following CLT

$$
\frac{\mathcal{L}_{d-k}\left(A_{u}(f ; \mathcal{T})\right)-\mathbb{E}\left[\mathcal{L}_{d-k}\left(A_{u}(f ; \mathcal{T})\right)\right]}{|\mathcal{T}|} \underset{\mathcal{T} \rightarrow \mathbb{R}^{d}}{\longrightarrow} N\left(0, \sigma_{d-k}^{2}(u)\right),
$$

where $\sigma_{d-k}^{2}(u)$ is given by $\sum_{q \geq 1} V_{q}^{k}$ in (41).

\section{Discussion}

\section{EXTENSION TO GENERAL PARAMETER SPACES:}

Notice that the only place where we required the box type shape of the parameter space is when we get an upper bound on the limiting variance of $\mathcal{L}_{d-k}^{\#}\left(A_{u}(f ; \mathcal{T})\right)$. However, this can be overcome by a limiting procedure.

Let us partition the space $\mathbb{R}^{d}$ into small cuboids of volume $\delta$. We can identify these small cuboids by the centre of the cuboids. Let $C_{T}^{\delta}$ be the set of cuboids which completely lie in the set $\mathcal{T}$, and $B_{\mathcal{T}}^{\delta}$ be the cuboids which have non empty intersection with he set $\mathcal{T}$ and the complement of $\mathcal{T}$.

Denoting $\mathcal{P}_{i, \delta}$ for the elements of the partition of $\mathbb{R}^{d}$ into cuboids of volume $\delta$, we have

$$
\begin{aligned}
\mathcal{L}_{d, d-k}^{\#}\left(A_{u}(f ; \mathcal{T})\right) & =\sum_{\mathcal{P}_{i, \delta} \in C_{\mathcal{T}}} \mathcal{L}_{d, d-k}^{\#}\left(A_{u}\left(f ; \mathcal{P}_{i, \delta}\right)\right)+\sum_{\mathcal{P}_{i, \delta} \in B_{\mathcal{T}}} \mathcal{L}_{d, d-k}^{\#}\left(A_{u}\left(f ; \mathcal{P}_{i, \delta}\right)\right) \\
& =\mathcal{L}_{d, d-k}^{\#}\left(A_{u}(f ; \mathcal{T}), 1\right)+\mathcal{L}_{d, d-k}^{\#}\left(A_{u}(f ; \mathcal{T}), 2\right) .
\end{aligned}
$$

Notice that using stationarity and the decay of covariance function $\gamma$, like in [6], we can conclude that

$$
\operatorname{var}\left(\mathcal{L}_{d, d-k}^{\#}\left(A_{u}(f ; \mathcal{T}), 1\right)\right)=O\left(\left|C_{\mathcal{T}}^{\delta}\right|\right)
$$

where $\left|C_{\mathcal{T}}^{\delta}\right|$ is the cumulative volume of al cuboids which constitute $C_{\mathcal{T}}^{\delta}$. Next, observe that $\left|C_{\mathcal{T}}^{\delta}\right| \rightarrow|\mathcal{T}|$ as $\delta \rightarrow 0$. Implying that the contribution by the boundary terms to the variance is $o(1)$, and thus can be ignored, which eventually means that the asymptotic Gaussianity can be proved by following the same methods as sketched out in this paper, when considering a $d$-dimensional compact, convex, symmetric ${ }^{4}$ subset of $\mathbb{R}^{d}$, as parameter space $\mathcal{T}$.

JOINT CONVERGENCE OF THE VARIOUS LKCS:

We note here that using similar ideas, one can prove the multivariate case for different values of the threshold $u$. One of the important question to look forward to, is the joint distribution of various LKCs evaluated at a fixed threshold. Though, the authors believe the joint convergence can be proven, but getting meaningful estimates on limiting covariances is likely to be challenging.

Nevertheless, it is worth noticing that the computations done to obtain Theorem 1.1 allow to get in a straightforward way a multivariate CLT for the $\operatorname{EPCs}\left(\mathcal{L}_{0}\left(A_{u}\left(f ; \mathcal{T}_{U_{i}}\right)\right), i=1, \cdots, n\right)$ :

\footnotetext{
${ }^{4}$ By a symmetric set, we mean that every chord passing through the origin must be bisected at the origin.
} 
Corollary 4.1 (ClT for multivariate $\left.\left(\mathcal{L}_{0}\left(A_{u}\left(f ; \mathcal{T}_{U_{i}}\right)\right), i=1, \cdots, n\right)\right)$

Under Hypothesis (H1) to (H3), we have, for any $U_{i} \in \operatorname{Graff}(d, k), i=1, \cdots, n$,

$$
\left(\mathcal{L}_{0}^{\#}\left(u, \mathcal{T} ; U_{i}\right), i=1, \cdots, n\right)^{t} \rightarrow N\left(0, \Sigma_{0, n}(u)\right), \text { as } \mathcal{T} \rightarrow \mathbb{R}^{d},
$$

with $\Sigma_{0, n}(u)=\left(\sigma_{0, U_{i}, U_{j}}(u)\right)_{1 \leq i, j \leq n}$ the limiting covariance matrix, $\sigma_{0, U_{i}, U_{j}}(u)$ being the limit, as $\mathcal{T} \rightarrow \mathbb{R}^{d}$, of the following

$$
\begin{aligned}
& \frac{1}{\left|\mathcal{T}_{U_{i}}\right|^{1 / 2}\left|\mathcal{T}_{U_{j}}\right|^{1 / 2}} \sum_{q=1}^{\infty} \sum_{|\underline{n}|=q} \sum_{|\underline{m}|=q} c(\underline{n}, u, k, \Lambda) c(\underline{m}, u, k, \Lambda) \\
& \times \sum_{\substack{d_{i j} \geq 0 \\
\sum_{i}^{i} d_{i j}=n_{j} \\
\sum_{j}^{j} d_{i j}=m_{i}}} \underline{n} ! \underline{m} ! \int_{x \in \partial_{k}} \int_{\mathcal{T}_{U_{i}} w \in \partial_{k}\left(x-\mathcal{T}_{U_{j}}\right)} \prod_{1 \leq i, j \leq D} \frac{\gamma_{i j}^{d_{i j}}(w)}{d_{i j} !} \mathcal{H}_{k}(d w) \mathcal{H}_{k}(d x) .
\end{aligned}
$$

Note that the variances are finite and positive. Moreover, the limiting covariance can be identified by observing that $\mathcal{L}_{0}\left(A_{u}\left(f ; \mathcal{T}_{U^{*}}\right)\right.$ for $U^{*} \in G r a f f(d, k)$ such that $0 \notin U^{*}$, is identical in distribution to $\mathcal{L}_{0}\left(A_{u}\left(f ; \widetilde{\mathcal{T}_{U^{*}}}\right)\right.$ due to isotropy, where $\widetilde{\mathcal{T}_{U^{*}}}$ is the parallel shift of $\mathcal{T}_{U^{*}}$ such that $0 \in \widetilde{\mathcal{T}_{U^{*}}}$.

Notice that convergence of finite dimensional vectors like above might help to obtain the CLT for general LKCs in an alternative way. If we could ensure the tightness, then applying the Hadwiger formula (7) would allow to conclude the CLT of $\mathcal{L}_{k}\left(A_{u}(f ; \mathcal{T})\right)$. Nevertheless, proving the tightness on such a space is still an open problem.

\section{Acknowledgments}

Both authors kindly acknowledge the financial support received from IFCAM (Indo-French Center for Applied Mathematics) to work on this project in India (TIFR - CAM, Bangalore) and in France (ESSEC Business school, Paris) in 2014 and 2015. This project has also received the support from the European Union's Seventh Framework Programme for research, technological development and demonstration under grant agreement no 318984 - RARE, and from the Airbus Foundation Chair on Mathematics of Complex Systems at TIFR-CAM, Bangalore. This study has been presented at EVA2015 (invited 'RARE' session on June 17, 2015; see http://sites.lsa.umich.edu/eva2015), then at the Monash Probability Conference in Honor of Robert Liptser in Prato (April 28, 2016; see http://monash.it/news-andevents/event/show/monash-probability-conference-honor-robert-liptsers-80th-birthday). 


\section{References}

[1] Adler, R. J. and Taylor, J. E. (2007) Random Fields and Geometry, Springer.

[2] Arcones, M. A. (1994) Limit Theorems for Nonlinea Functionals of a Stationary Gaussian Sequence of Vectors. Ann. Probab. 22, no. 4, 2242-2274.

[3] Azaïs, J. M. and Wschebor, M. (2009) Level sets and Extrema of Random Processes and Fields, Wiley.

[4] Berman S. (1991) Sojourns and Extremes of Stochastic Processes, Wadsworth \& Brooks.

[5] Blaszczyszyn, B., Yogeshwaran, D. and Yukich, J. E. (2016) Limit theory for geometric statistics of clustering point processes. arXiv:1606.03988

[6] Estrade, A. and León, J. (2016) Euler characteristic of excursion of Gaussian random fields. Ann. Probab. (forthcoming).

[7] Geman, D. (1972) On the variance of the number of zeros of a stationary Gaussian process. Ann. Math. Stat., 43, 977-982.

[8] Giraitis, L. and Surgailis, D. (1985) CLT and other limit theorems for functionals of Gaussian processes. Z. Wahrsch. verw. Geb., 70, 191-212

[9] Hotelling, H. (1939) Tubes and Spheres in $n$-Spaces and a Class of Statistical Problems, Amer. J. Math., 61: 440-460.

[10] Klain, D. and Rota, G-C. (2008) Introduction to Geometric Probability, Lezioni Lincee, Cambridge University Press

[11] Kratz, M. (2006) Level crossings and other functionals of stationary Gaussian processes, Probability Surveys, 3, 230-288.

[12] Kratz, M. and León, J. R. (1997) Hermite polynomial expansion for non-smooth functionals of stationary Gaussian processes: crossings and extremes, Stoch. Proc. App. $66,237-252$.

[13] Kratz, M. and León, J. R. (2000) Central limit theorems for the number of maxima and some estimator of the second spectral moment pf a stationary Gaussian process with an application in hydroscience, Extremes 3:1, 57-86.

[14] Kratz, M. and León, J. R. (2001) Central limit theorem for level functionals of stationary Gaussian processes and fields. J. Theor. Probab. 14(3), 639-672.

[15] Kratz, M and León, J. R. (2006) On the second moment of the number of crossings by a stationary Gaussian process. Ann. Probab. 34, No. 4, 1601-1607.

[16] Marinucci, D. and Cammarota, V. (2016) A Quantitative Central Limit Theorem for the Euler-Poincar Characteristic of Random Spherical Eigenfunctions. arXiv:1603.09588 
[17] Marinucci, D. and Vadlamani, S. (2016) High-frequency asymptotics for LipschitzKilling curvatures of excursion sets on the sphere. Ann. Appl. Probab. 26, No.1, $462-506$.

[18] Meshenmoser, D. and Shashkin, A. (2011) Functional central limit theorem for the volume of excursion sets generated by associated random fields. Stat. Probable. Lett. Vol. 81, No. 6.

[19] Nourdin, I. and Peccati, G. (2012) Normal Approximations Using Malliavin Calculus: from Stein's Method to Universality, Cambridge University Press.

[20] Nourdin, I., Peccati, G. and Podolskij, M. (2011) Quantitative Breuer-Major Theorems. Stoch. Proc. Appl. 121(4), 793-812.

[21] Nualart, D. (2006) The Malliavin calculus and related topics, Springer-Verlag, Heidelberg.

[22] Pham, V.-H. (2013) On the rate of convergence for central limit theorems of sojourn times of Gaussian fields. Stoch. Proc. Appl. 123(6), 2158-2174.

[23] Slud, E. (1991) Multiple Wiener-Itô integral expansions for level-crossing-count functionals. Probab. Th. Rel. Fields 87, 349-364.

[24] Slud, E. (1994) MWI representation of the number of curve-crossings by a differentiable Gaussian process, with applications. Ann. Probab. 22, no. 3, 1355-1380.

[25] Spodarev, E. (2013) Limit theorems for excursion sets stationary associated random fields. Chapter in Modern Stochastics and Applications, Vol. 90 of the series Springer Optimization and Its Applications, 221-241.

[26] Szegö, G (1975) Orthogonal polynomials, 4th edition, American Mathematical Society, Colloquium Publications, Vol. XXIII. American Mathematical Society, Providence, R. I.

[27] Taqqu, M. (1977) Law of Iterated Logarithm for Sums of Non-Linear Functions of Gaussian Variables that Exhibit a Long Range Dependence. Z. Wahrsch. verw. Geb. 40, 203-238.

[28] Weyl, H. (1939), On the Volume of Tubes, Amer. J. Math., 61: 461-472

[29] Wschebor, M. (1985) Surfaces aléatoires. Mesure géométrique des ensembles de niveau. L.N.M. 1147, Springer. 
ESSEC Business School

3, avenue Bernard-Hirsch

CS 50105 Cergy

95021 Cergy-Pontoise Cedex

France

Tél. + $33(0) 134433000$

www.essec.edu
ESSEC Executive Education

CNIT BP 230

92053 Paris-La Défense

France

Tél. +33 (0) 146924900

www.executive-education.essec.fr
ESSEC Asia-Pacific

5 Nepal Park

Singapore 139408

Tél. +65 68849780

www.essec.edu/asia

\section{SINGAPOUR}

Contact :

Centre de Recherche

+33 (0)134433091

research.center@essec.fr

ISSN 1291-9616 